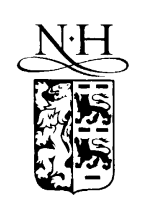

ELSEVIER

\title{
Morphology of ultrathin Ag films grown on Mo(llll)
} \author{
M.-T. Lin ${ }^{\mathrm{b}}$ \\ ${ }^{a}$ Institute of Atomic and Molecular Sciences, Academia Sinica, P.O. Box 23-166, Taipei, 10764, Taiwan, ROC \\ ${ }^{\mathrm{b}}$ Department of Physics, National Taiwan University, Taipei, Taiwan, ROC \\ Received 15 June 2000; accepted for publication 4 January 2001
}

\begin{abstract}
Ultrathin films of Ag grown on Mo(1 11 ) and vicinal surfaces have been studied using temperature programmed Auger, LEED and thermal desorption spectroscopy. We confirmed that Ag does not induce faceting of Mo(1 111$)$ and growth of Ag follows the Stranski-Krastanov mode as reported previously. In addition, we found that (1) on top of the wetting layer; there is a two-dimensional (2-d) gas-like phase in coexistence with the Ag 3-d clusters. The density of this gas increases with temperature and can reach a very high value of about 0.8 monolayer at $900 \mathrm{~K}$. (2) 2-d films deposited at $120 \mathrm{~K}$ show an anomalous coverage dependent stability against 3-d cluster formation. (3) If the total coverage is not more than 0.3 monolayer above the wetting layer, the film deposited at low temperature is unstable and 3-d clusters readily form when annealed around $450 \mathrm{~K}$. However, once annealed to $750 \mathrm{~K}$, the 3-d clusters fully re-spread and the 2-d film formed is trapped in a supersaturated state, i.e. although its coverage is larger than the wetting layer, cluster formation no longer occurs whatever way we anneal it. Auger and LEED evidences suggest that films annealed above 600 $\mathrm{K}$ undergo interface confined mixing of $\mathrm{Ag}$ and Mo. (4) On the vicinal surface we studied, although nucleation of 3-d clusters can occur at a temperature $50 \mathrm{~K}$ lower than that on the singular surface, the density of the 2-d gas-like phase on the vicinal surface is basically the same as on the singular surface. (c) 2001 Elsevier Science B.V. All rights reserved.

Keywords: Surface thermodynamics (including phase transitions); Faceting; Thermal desorption; Wetting; Auger electron spectroscopy; Molybdenum
\end{abstract}

\section{Introduction}

The bimetallic system of $\mathrm{Ag} / \mathrm{Mo}\left(\begin{array}{lll}1 & 1 & 1\end{array}\right)$ is of interest for two reasons. First, it has been found experimentally that the capability of different adsorbates to induce facet formation of W(1 111$)[1,2]$ and $\operatorname{Mo}(111)$ [3] is correlated to the Pauling

\footnotetext{
${ }^{*}$ Corresponding author. Tel.: +886-2-23668262; fax: +886-223620200.

E-mail address: song@po.iams.sinica.edu.tw (K.-J. Song).
}

electronegativity (PE) of the adsorbates. It has been further proposed [4] that the PE of the adsorbate be correlated with the adsorbate enhanced surface energy anisotropy of $\operatorname{Mo}(111)$, which drives the facet formation process if the enhanced anisotropy is large enough. However, more recent photoemission study [5] indicates there is no clear correlation between interface $\mathrm{W} 4 \mathrm{f}_{7 / 2}$ binding energy and PE. Thus, it remains a mystery how the electronegativity gets into play. $\mathrm{Au}$ and $\mathrm{Ag}$ are adsorbates of rather similar chemical property and nearly identical lattice size but very different PE 
(2.4 for $\mathrm{Au}$ and 1.9 for $\mathrm{Ag}$ ). Thus they should be the most interesting pair of adsorbates for the purpose of addressing the potential role played by the electronegativity. Theoretically, the embedded atom method [6] as well as first-principles ab initio calculations [7] have been applied and are quite successful in explaining the behavior of many of these bimetallic systems. One example is the explanation [8] of the recent finding that in addition to inducing $\left\{\begin{array}{lll}1 & 1 & 2\end{array}\right\}$-oriented facets, Pd also induces $\left\{\begin{array}{ll}1 & 0\end{array}\right\}$-oriented facets on W(1 11 ). Although successful in predicting many individual cases, so far the calculations have not generated a new hypothesis about general guideline or trend such as the electronegativity correlation. In fact, one of the calculations in Ref. [7] may provide a counter example of the electronegativity correlation. According to Ref. [7], when covered with one physical monolayer of Ag, the $\left\{\begin{array}{lll}1 & 1 & 2\end{array}\right\}$-faceted form could be lower in energy than the planar $1 \times 1$ form and facet formation could occur for $\mathrm{Ag} /$ Mo(1 11 1). Experimentally, Ref. [3] reported that the $\mathrm{Ag} / \mathrm{Mo}\left(\begin{array}{lll}1 & 1 & 1\end{array}\right)$ surface remains planar. The authors in Ref. [7] proposed that this apparent disagreement could be due to a high nucleation barrier for facet formation. Given the importance of the $\mathrm{Ag} / \mathrm{Mo}\left(\begin{array}{lll}1 & 1 & 1\end{array}\right)$ case, we re-examined the $\mathrm{Ag} /$ Mo(1 111 ) system and confirmed that Ag does not induce faceting of $\operatorname{Mo}(111)$ if deposited and annealed the usual way. However, we find the Ag/ $\operatorname{Mo}\left(\begin{array}{lll}1 & 1 & 1\end{array}\right)$ to assume several planar structures with large superlattices that have not been reported before. As these large planar super-structures have not been considered in the calculation of Ref. [7], the possibility that some of these planar forms have lower surface energy than the faceted form cannot be ruled out completely and the electronegativity correlation still have a chance to survive.

There is another interesting aspect about $\mathrm{Ag}$ films grown on $\operatorname{Mo}(111)$. Although no evidence of Ag induced faceting of either W(1 111$)$ or Mo(1 111$)$ has been found, Ref. [3] did report an interesting phenomenon. On both surfaces, growth of ultrathin Ag films follows the Stranski-Krastanov mode, i.e. formation of three-dimensional (3-d) clusters after one wetting layer. However, these two systems are unusual as the 3-d clusters par- tially re-spread onto the surface after being annealed above $800 \mathrm{~K}$. Note that Ag films grown on (110) surfaces of III-V compound semiconductors and $\mathrm{Si}\left(\begin{array}{lll}1 & 1 & 1\end{array}\right)-7 \times 7$ [9] are also known to have metastable planar structures which are believed to be stabilized by electronic contributions to the free energy [10]. Given the general interest in factors that control and/or modify the growth mode of hetero-epitaxy systems, re-spreading of $\mathrm{Ag}$ on $\mathrm{Mo}\left(\begin{array}{lll}1 & 1 & 1\end{array}\right)$ and $\mathrm{W}\left(\begin{array}{lll}1 & 1 & 1\end{array}\right)$ is certainly worth a more detailed examination. In this paper, we will show that re-spreading of Ag 3-d clusters on $\mathrm{Mo}\left(\begin{array}{lll}1 & 1 & 1\end{array}\right)$ is due to: (1) evaporation into a high density 2 -d gas at elevated temperature and (2) remaining in a supersaturated 2-d states when the sample is cooled. Trapping in a supersaturated state is possible only when there are very few or no 3 -d clusters left on the surface.

\section{Experiment}

The experiment is performed in a UHV chamber with base pressure of $6 \times 10^{-11}$ Torr. This chamber is equipped with three analytical tools: (a) a PHI 10-155 cylindrical mirror analyzer (CMA) with coaxial electron gun for Auger Spectroscopy, (b) a VG RVL900 4-grid low energy electron diffraction (LEED) system augmented with a CCD camera, and (c) an UTI 100C quadruple mass analyzer. The molybdenum single crystals are provided by Metal Crystals \& Oxides Ltd. with a specified mosaic spread of less than $0.5^{\circ}$. They are cut within $0.5^{\circ}$ of the (1 111 ) direction and are $3 \mathrm{~mm}$ wide, $12 \mathrm{~mm}$ long and $0.4 \mathrm{~mm}$ thick. We electropolished the crystals down to a thickness of about $0.3 \mathrm{~mm}$ to get a smooth surface. Although we always get a smooth surface, one of the crystals became more rounded than the others. On this crystal, we find it possible to locate regions of singular (1 111 ) surface and regions of vicinal surface with a $3.5^{\circ}$ angle of miscut toward [1 $\left.11-2\right]$ direction. This facilitates study of effect of steps oriented along $[-110]$ direction. Sample temperature is measured by either a C-type thermocouple spot welded at the center of the back of the crystal or a pair of Ta wires spot welded at two points 
$6 \mathrm{~mm}$ apart at the back of the crystal to pickup the potential drop due to resistive heating. The sample and its holder are attached to the end of a liquidnitrogen Dewar for cooling. The sample is heated by passing a current through the sample. Heating current is provided by a programmable power supply, which is controlled by a personal computer to facilitate programmed heating. To enable distortion free Auger or LEED detection during programmed heat treatment, the control signal (and thus the heating current) can be chopped off by a $50 \mathrm{~Hz}$ periodic gating signal. Distortion free detection of Auger or LEED signal then proceeds during the gate period when the heating current is off. Sample cleaning is by repetitive dosing of oxygen and flashing up to $2100 \mathrm{~K}$. This procedure is carried out many times until carbon segregation onto the surface is no longer detectable by Auger after the sample is annealed at $2100 \mathrm{~K}$ for $30 \mathrm{~s}$.

Deposition of high purity (99.9985\%) Ag is from a home made temperature programmed sublimator. Once cleaned, the pressure rise (primarily due to $\mathrm{CO}$ ) during evaporation is less than $1 \times 10^{-10}$ Torr at a deposition rate of one hundredth of a monolayer per second $(0.01 \mathrm{ML} / \mathrm{s})$. By varying the programmable source temperature and heating time, different deposition rate and total amount can be obtained. At a deposition rate of one hundredth of a monolayer per second, the coverage can be controlled to a precision of about one fortieth of a monolayer.

\section{Results}

\subsection{Relative coverage calibration points and appar- ent thickness of Ag films}

Curves shown in Fig. 1 are a series of temperature programmed thermal desorption traces of $\mathrm{Ag}$ from Mo(1 11 ) surface. All traces have the same constant heating rate of $10 \mathrm{~K} / \mathrm{s}$, but with different initial coverage. As the Ag coverage increases, desorption gradually shifts toward lower temperature side until the curve labeled P. Further increase of $\mathrm{Ag}$ coverage caused emergence of a new desorption peak which is characterized by a common leading edge (L) and peak positions that

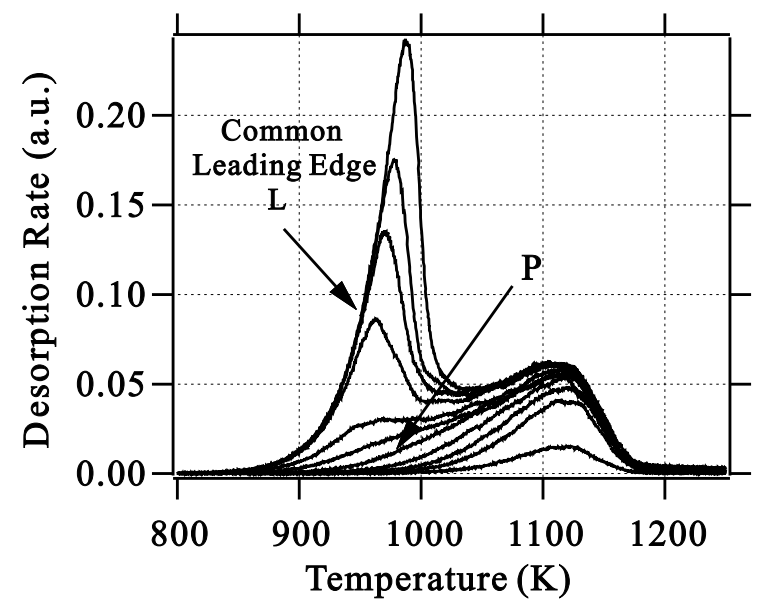

Fig. 1. A series of temperature programmed thermal desorption traces of $\mathrm{Ag}$ from $\mathrm{Mo}(111)$ surface. All traces have the same heating rate of $10 \mathrm{~K} / \mathrm{s}$, but with different initial coverage. We define the coverage of the surface that give rise to the desorption trace labeled $\mathrm{P}$ to be one TDML.

shift toward higher temperatures with larger coverage. These are features of zero order desorption kinetics and indicative of formation of multilayer Ag films. Thus, we define the coverage of the surface that give rise to the desorption trace labeled P to be one "thermal desorption monolayer" (TDML). We will show in Section 3.2 that 1 TDML approximately equals to one Ag atom per exposed substrate Mo atom. Throughout this paper, if we do not explicitly state otherwise, monolayer means TDML.

The Auger peak to peak amplitudes of $\mathrm{Ag}$ (at $351 \mathrm{eV}$ ) and Mo (at $186 \mathrm{eV}$ ) are measured for a series of $\mathrm{Ag}$ films that is deposited and held below 120 K. As shown in Fig. 2, both can be fit with the following exponential forms:

$$
\operatorname{Amp} 351=a^{*}[1-\exp (-\theta / \Delta)]+b^{*} \exp (-\theta / \Delta)
$$

$\operatorname{Amp} 186=c^{*} \exp (-\theta / \delta)+d^{*}[1-\exp (-\theta / \delta)]$.

By normalizing to the Auger strength of Mo at 186 $\mathrm{eV}$, we have $a=3.66, b=3.34 \times 10^{-3}, c=1$, and 


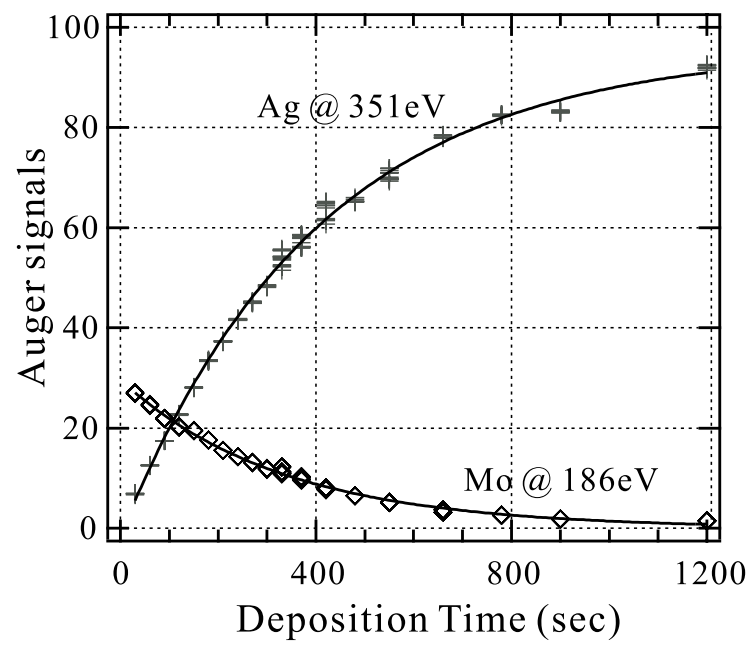

Fig. 2. Auger strength of $\mathrm{Ag}$ and Mo for a series of $\mathrm{Ag}$ films on Mo(1 11 1) deposited at and held below $120 \mathrm{~K}$. Plus (for Ag) and diamonds (for Mo) are raw data, while lines are the best fitting exponential forms.

$d=1.1 \times 10^{-3} . a$ is the Auger strength of $\mathrm{Ag}$ at $351 \mathrm{eV}$ for very thick Ag film, $b$ is the small Auger signal of Mo at $351 \mathrm{eV}$, and $d$ is the small Auger contribution of $\mathrm{Ag}$ at $186 \mathrm{eV} . \Delta$ is the effective decay constant at $351 \mathrm{eV}$, which is found to be $1.90 \pm 0.05$ TDML $\delta$ is the decay constant at 186 $\mathrm{eV}$, which is found to be $1.57 \pm 0.03$ TDML. Such exponential behavior suggests that at or below 120 $\mathrm{K}$, Ag films undergo simultaneous multilayer growth and should be "statistically uniform". For such films, the ratio of Auger amplitude at $351 \mathrm{eV}$ to that at $186 \mathrm{eV}$ is:

$$
\begin{aligned}
R(\theta)= & \left\{a^{*}[1-\exp (-\theta / \Delta)]+b^{*} \exp (-\theta / \Delta)\right\} \\
& /\left\{c^{*} \exp (-\theta / \delta)+d^{*}[1-\exp (-\theta / \delta)]\right\} .
\end{aligned}
$$

Since this ratio $R(\theta)$ is a monotonic increasing function of coverage, the inverse function $\theta(R)$ can be constructed. Then, for "statistically uniform" films, we can find the real coverage from the measured ratio by applying the inverse function $\theta(R)$. For any arbitrary Ag film, we can still measure the ratio $R$ and define the "apparent thickness" of such a film to be $\theta(R)$. We will make use of this "apparent thickness" in presenting most of our Auger results.

\subsection{Planar superstructures of the $A g / M o(111)$ system}

In all our LEED experiments, the $\operatorname{Mo}\left(\begin{array}{lll}1 & 1 & 1\end{array}\right)$ surface is always below $120 \mathrm{~K}$ when we deposit Ag. After deposition, the surface is annealed at various temperatures starting at $150 \mathrm{~K}$ with $50 \mathrm{~K}$ interval up to $800 \mathrm{~K}$. After each annealing process, we observe the LEED pattern when the sample temperature is cooled down below $120 \mathrm{~K}$. After the LEED observations are done, we perform temperature programmed thermal desorption. Desorption of both Ag and carbon monoxide [11] (the major contaminant) is recorded simultaneously. The sample is then flashed clean for a new experiment. By integrating the desorption traces of $\mathrm{CO}$, we found that for surfaces with sub-monolayer Ag coverage, the amount of CO clearly correlated with the duration of time spent on the LEED observations, and can reach $5 \%$ of CO's saturation coverage on clean $\operatorname{Mo}(111)$ in the worst case. Once the Ag coverage is above 1 TDML, the amount of $\mathrm{CO}$ found is always below a few thousandths of CO's saturation coverage and independent of the amount of time the sample has stayed in the chamber. Obviously, once covered with $\mathrm{Ag}$, the surface is passivated. Thus, for films with coverage above one TDML, we can do prolonged experiments with them without problems of contamination. For films with sub-monolayer of $\mathrm{Ag}$, we double-checked those LEED patterns obtained after prolonged annealing processes by observing samples annealed immediately after the deposition. Except for some patterns being more vague, most LEED patterns obtained the fast way show no qualitative difference from those obtained the slow way. Thus, although the contaminating $\mathrm{CO}$ may cause some perturbation of the superstructure, their influence on the superstructure seems negligible.

Surfaces with 36 different coverages of $\mathrm{Ag}$ (ranging from clean to 3 TDML) have been studied and no evidence of facet formation has ever been found, which is in accord with Ref. [3]. As a function of both coverage and annealing temperature, we do find many different superstructures for $\mathrm{Ag} / \mathrm{Mo}\left(\begin{array}{lll}1 & 1 & 1\end{array}\right)$. Some characteristic LEED patterns are shown in Fig. 3. All samples have been 

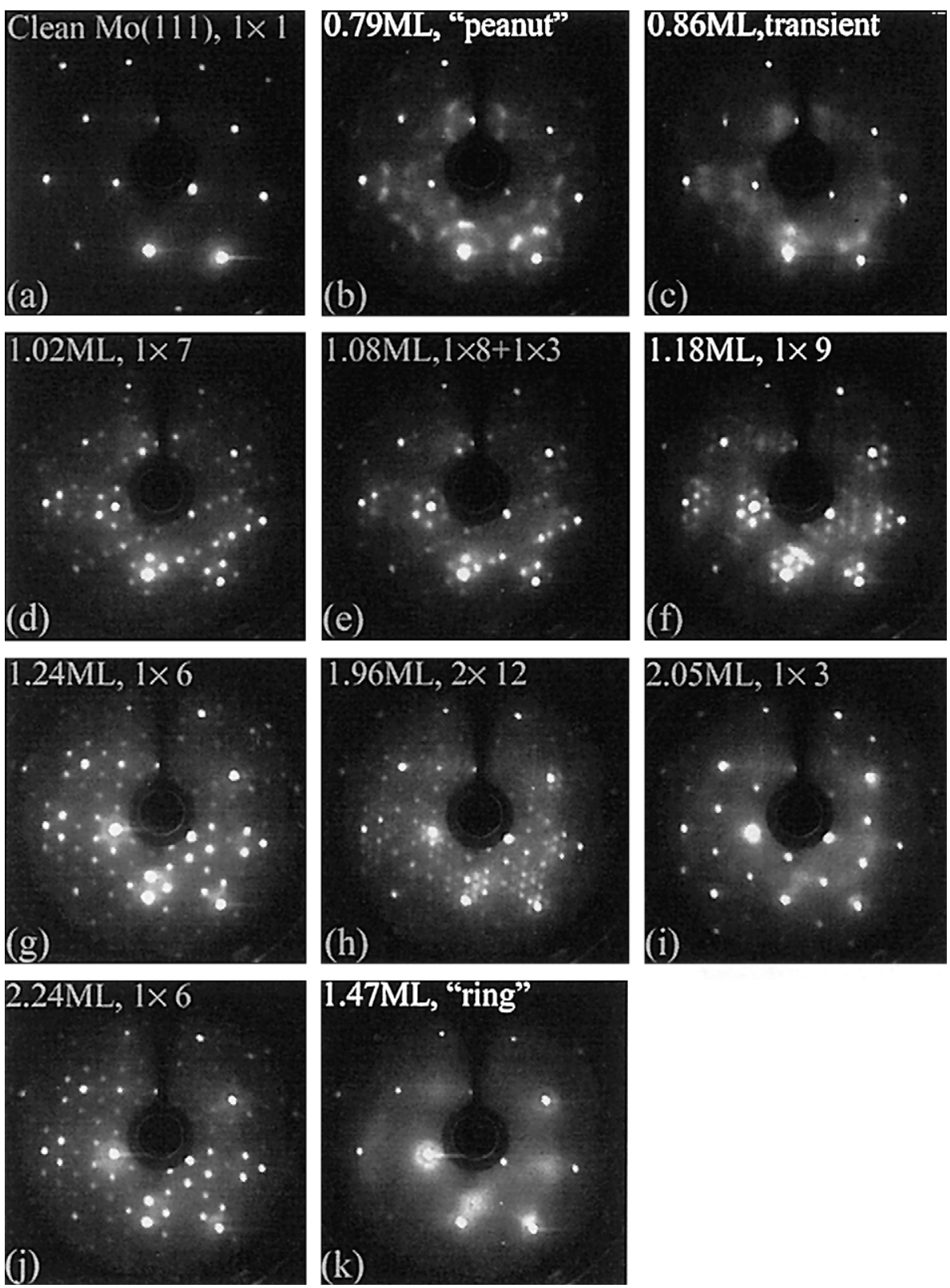

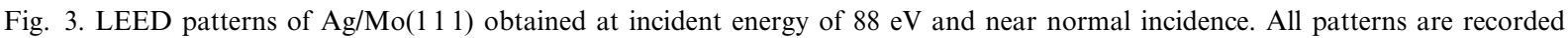
while the sample is at temperatures below $120 \mathrm{~K}$. All samples have been annealed up to $350 \mathrm{~K}$ except pattern (i) and (k), which have been annealed up to 200 and $550 \mathrm{~K}$ respectively. For our notation, ML refers to TDML. For $m \times n$ structure, the real space primitive basis vectors of the superlattice cells are $m^{*} a(1,1,-2)$ and $n^{*} a(1,0,-1)$, where $a$ is the lattice constant of Mo. Since Mo(11 1) surface has three fold rotational symmetry, the two other equivalent primitive basis set can be obtained by rotating the three $(x, y, z)$ coordinates clockwise. We also note that our $1 \times 3$ is the same as the more commonly known $\sqrt{ } 3 \times \sqrt{ } 3 \mathrm{R} 30^{\circ}$.

annealed up to $350 \mathrm{~K}$ except (i) and (k), which have been annealed up to 200 and $550 \mathrm{~K}$ respec- tively. We note in particular that near a coverage of one TDML the surface assumes a series of super 
structures changing from $1 \times 7$, a mixture of $1 \times 8$ plus $1 \times 3,1 \times 9$, and finally a $1 \times 6$ structure. For coverage larger than 1.16 TDML, additional Ag tend to form 3-d clusters when annealed. The $1 \times 7$ and $1 \times 6$ structures are of particular interest. The $1 \times 7$ is the structure of the film when the coverage is $1.0 \mathrm{TDML}$ while the $1 \times 6$ is the structure of the wetting layer if the annealing temperature is below $550 \mathrm{~K}$. As there are three exposed substrate atoms in each $1 \times 1$ primitive cell, there are 21 (or 18) exposed substrate atoms in one $1 \times 7$ (or $1 \times 6$ ) super-cell . Assume there are $N \mathrm{Ag}$ atoms in one $1 \times 7$ supercell and $M \mathrm{Ag}$ atoms in one $1 \times 6$ supercell. As the coverage of the wetting layer is 1.16 TDML, this means $M=N$. Estimation based on Auger decay length [12] further suggest that $N$ is in the range from 17 to 21 . Thus, 1 TDML approximately equals to one (or slightly less) Ag atom per exposed substrate Mo atom. In particular, we note that thermal desorption of a film with the coverage of the wetting layer shows a clear lower temperature shoulder, indicating that binding of some of the Ag atoms of the wetting layer are significantly weaker than others.

The calculation in Ref. [7] assumed $1 \times 1$ structure for both (1 111$)$ and (1 112$)$ surfaces. Since all the $1 \times n(1 \times 6$ in particular $)$ superstructure should be lower in energy than the $1 \times 1$ structure, it would be interesting to know how much is the energy difference between the theoretically best $1 \times$ 1 and the theoretically best $1 \times n$ (in particular $1 \times 6)$ superstructures. That may help us address whether the $1 \times 6$ structure can stabilize the (1 111$)$ surface against formation of $\{112\}$-oriented facets. We note in particular that in the case of $\mathrm{Au} /$ Mo(1 11 ), the Rutgers group observed facet formation [3] while our group [4] observed planar surface with a large superstructure. Although it is not yet known what caused the difference, it has been suggested [4] that the observed large superstructure may be responsible for stabilizing the $\mathrm{Au} / \mathrm{Mo}\left(\begin{array}{lll}1 & 1 & 1\end{array}\right)$ surface against facet formation.

Although each pattern shown in Fig. 3 is marked with a coverage, it is not implied that there is only one characteristic structure at any coverage. On the contrary, the superstructure observed depends on the highest temperature the sample has been annealed at and usually there is more than one intermediate structure. For all surfaces with Ag coverage larger than $0.3 \mathrm{TDML}$, once the sample is annealed above $550 \mathrm{~K}$, all superlattice spots disappear even after the sample cools down and the LEED pattern become like that shown in Fig. 3(k). This LEED pattern is characterized by cloudy rings and blurred patches in addition to well-defined $1 \times 1$ spots. Major feature is the bright ring surrounding the $(0,0)$ order specular beam. The radius of the ring (in $k$ space) is coverage dependent, ranging from about 0.33 to 0.26 $\AA^{-1}$, being slightly larger at lower coverage. In real space this would correspond to a characteristic length of 19 to $24 \AA$. This "ring phase" should be the thermodynamically most stable phase as prolonged annealing at lower temperature cannot cause re-appearance of any of the $1 \times n$ superstructure. We will further discuss the nature of the ring phase later. Here, we just note that it seems well beyond the capability of current ab initio calculations to correctly predict such disordered structures.

\subsection{Re-spreading of 3-d clusters}

Using gated heating and gated Auger detection, we can obtain real time information of the evolution of the film while it is being annealed. Fig. 4(a) shows the raw data of a typical experiment as a function of time. 4 TDML of $\mathrm{Ag}$ had been deposited with the $\operatorname{Mo}\left(\begin{array}{lll}1 & 1 & 1\end{array}\right)$ surface held below 120 $\mathrm{K}$. The red curve shows the sample temperature as a function of time. In this particular experiment, whenever the sample temperature is raised, it is increased at $2 \mathrm{~K} / \mathrm{s}$ up to $850 \mathrm{~K}$. The sample temperature is then lowered in several different ways: 1 $\mathrm{K} / \mathrm{s}$, quenching, holding for $60 \mathrm{~s}$ at $850 \mathrm{~K}$ followed by quenching, and $1 \mathrm{~K} / \mathrm{s}$ again. Also plotted are the Auger peak to peak amplitudes of Ag (blue) and Mo (green). The two major features of interest are clearly observable. The first major feature is the irreversible drop of Ag Auger signal when its temperature is raised above $300 \mathrm{~K}$ the first time the sample is annealed after deposition. Such drop and the corresponding increase of Mo Auger signal is associated with the commonly observed phenomena of formation of 3-d clusters and will be discussed in more detail later. 

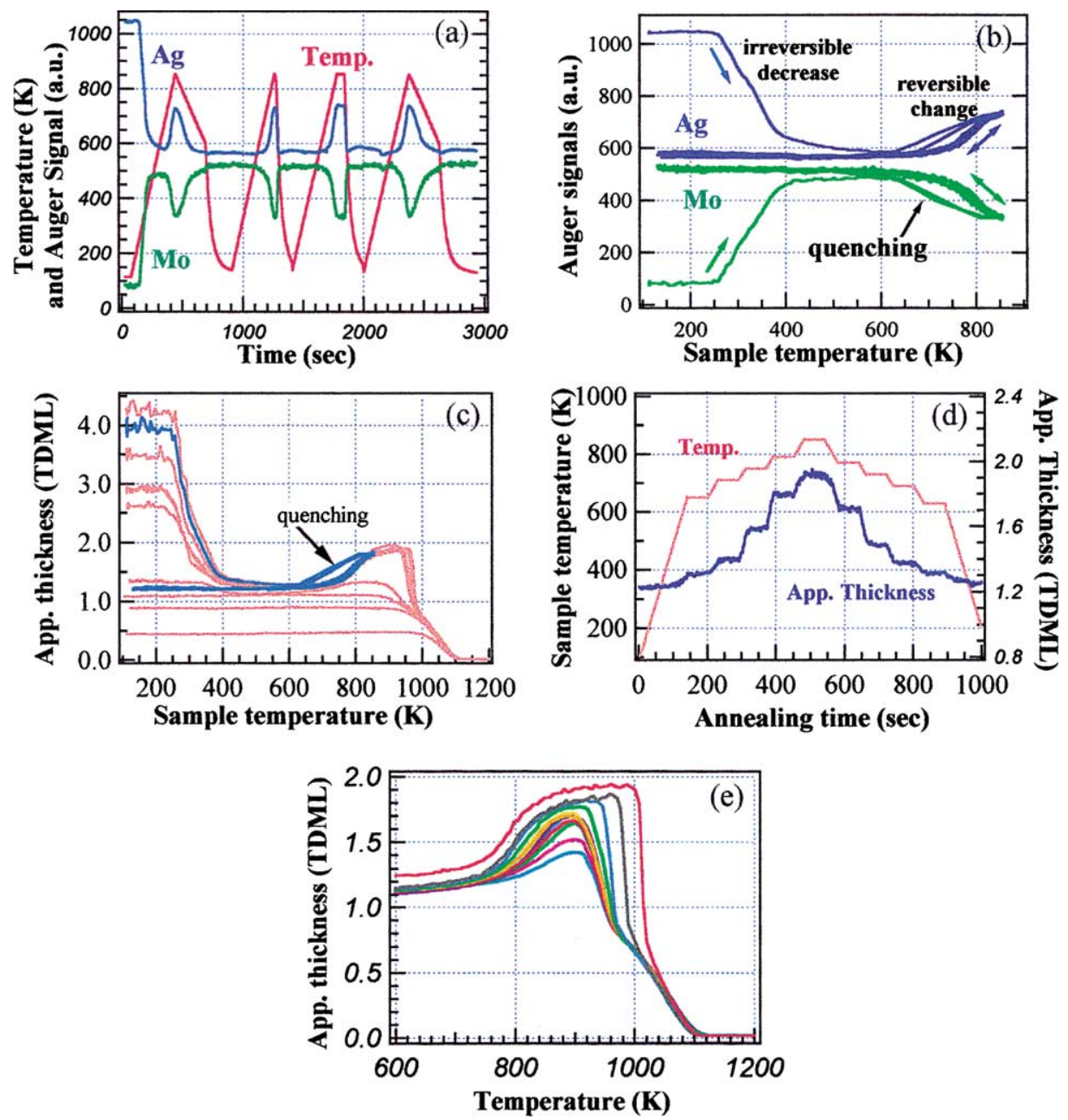

Fig. 4. (a) Auger signals and sample temperature as a function of time for an example experiment. (b) The same data but with Auger signals plotted as a function of sample temperature. (c) "Journey map" of the same experiment (blue) with apparent thickness plotted as a function of temperature. Pink background curves are apparent thickness of various films as a function of temperature when heated at $2 \mathrm{~K} / \mathrm{s}$ all way up to $1200 \mathrm{~K}$. (d) The temperature (red) and apparent thickness (blue) of a sample as a function of annealing time. Staircase like temperature programming is used to reveal the fast and reversible response of the film. (e) Apparent thickness for temperature larger than $600 \mathrm{~K}$ for another set of films when the films are heated all way up to 1200 at $2 \mathrm{~K} / \mathrm{s}$ rate.

The second feature is the unusual gradual increase (decrease) of $\mathrm{Ag}$ (Mo) Auger signal when the sample temperature is raised above $650 \mathrm{~K}$. The increase of Ag Auger accompanied by the decrease of Mo Auger certainly indicates that Ag from the 3 -d clusters has re-spread onto the surface. Fig. 4(a) shows degree of such re-spreading to be not only temperature dependent but also reversible. When the sample is cooled down slowly $(-1 \mathrm{~K} / \mathrm{s})$,
Ag Auger signal goes "down hill" following essentially the same "path" when it came uphill. This is better seen in Fig. 4(b), where the Auger signals are plotted as a function of sample temperature. In Fig. 4(b), except for the two quenching cases, all other curves fall onto one "beaten path".

From both Fig. 4(a) and (b), it is obvious that other than their change in opposite directions, the change of Mo Auger signal is a replica of the 
change of Ag Auger signal. Such "symmetry" is seen in all of our Auger data. Clearly, the same physical change is being reflected in both data. Here, the "apparent thickness" comes in handy not only for removal of redundancy but also for providing a linear measure of the average coverage of Ag as seen by Auger. In particular, we note that for films sparsely dotted with 3-d clusters, if these clusters only cover a negligible fraction of the surface area, then their contribution to the final Auger signal is also negligible. In such cases, the apparent thickness is a very faithful measure of the average thickness of those parts of the film not covered by the $3-d$ clusters.

In Fig. 4(c), the blue curve shows the "apparent thickness" of Ag plotted as a function of temperature for the same experiment as shown in Fig. 4(a) and (b). In this plot, it is now apparent that the initial coverage of the film is 4 TDML, and it is stable up to about $250 \mathrm{~K}$. Once annealed above $250 \mathrm{~K}$, about $3 \mathrm{TDML}$ of Ag are absorbed into the 3-d clusters and the film's apparent thickness decreases to about 1.2 TDML. Above $700 \mathrm{~K}$, the apparent thickness increases again with temperature and reaches a maximum of about 1.8 TDML at $850 \mathrm{~K}$.

The background red curves in Fig. 4(c) are the apparent thickness for a series of $\mathrm{Ag}$ films as a function of temperature when these films are being heated at $2 \mathrm{~K} / \mathrm{s}$ all way up to $1200 \mathrm{~K}$. For films with initial coverage less than 1.1 TDML, although our LEED observation indicates a lot of different superstructures depending on coverage and annealing history, nothing noticeable happens to the apparent thickness until $850 \mathrm{~K}$. This is not surprising, as the apparent thickness is supposedly not sensitive to the microscopic structural change of Ag parallel to the surface. Above $850 \mathrm{~K}$, thermal desorption of $\mathrm{Ag}$ is no longer negligible and caused the decrease of the apparent thickness all way to zero at about $1100 \mathrm{~K}$.

For films with initial coverage above 2.5 TDML, they all show signs of 3-d cluster formation at about room temperature. Above $700 \mathrm{~K}$, the apparent thickness of all these films increased in essentially the same way as the blue curve, irrespective of their initial coverage. This increase must be due to re-spreading of $\mathrm{Ag}$ from the 3-d Ag clusters out onto the surface again. Note that the degree of re-spreading is continuously variable and is an increasing function of temperature. To emphasize the fact that the surface is in equilibrium, results of an experiment with staircase like temperature-programmed heating and cooling is shown in Fig. 4(d). The red curve is the sample temperature while the blue curve is the apparent thickness of the Ag film. The sample of 6 TDML initial coverage had been annealed before and most of the Ag has already been incorporated into 3 -d clusters before this experiment. Clearly, whenever the sample heating or cooling is interrupted temporarily and held at a certain temperature, the apparent thickness also becomes constant, indicating that the film's apparent thickness follows closely the change in temperature when heated at 2 $\mathrm{K} / \mathrm{s}$. This strongly suggests that the change of apparent thickness be due to change of equilibrium condition. However, it is also clear from Fig. 4(b) that when the film is quenched (with initial cooling rate of $14.4 \mathrm{~K} / \mathrm{s}$ ), the film's response lags behind, indicating a time of several tens of seconds needed for adjusting to the new equilibrium condition. Nevertheless, attempts to freeze the Ag film in its high temperature re-spread state are not successful. Once the temperature decreases below about $600 \mathrm{~K}$, there is no difference in apparent thickness between slowly cooled and quenched $\mathrm{Ag}$ films. This is in apparent contradiction with Ref. [3], which observed re-spreading after the sample has been cooled down. We will resolve this issue later.

Coming back to Fig. 4(c), we note that for temperatures in between 850 and $930 \mathrm{~K}$, the increase of the apparent thickness slows down and saturates at about 1.9 TDML. Much more detail can be seen in Fig. 4(e). Why does the density of the re-spread film saturate at about 1.9 TDML? One possible reason for the density of the respread film to saturate at about 1.9 TDML is that the density may be limited by loss of Ag due to thermal desorption. As can be seen in Fig. 1, the desorption rate of $\mathrm{Ag}$ is significant in this temperature range. Only when the surface diffusion is very fast can the desorption loss be effectively replenished. Since the activation energy of surface diffusion is typically [13] from $10 \%$ to $50 \%$ that of 
the activation energy of thermal desorption, surface diffusion should dominates at low temperature. However, as temperature increases, the relative significance of thermal desorption will increase. Also, note that although depletion by desorption occurs uniformly across the re-spread film, the effectiveness of replenishing by surface diffusion depends strongly on how close the nearest 3-d cluster is. Thus, at high enough temperature and/or at places far away from any one of the 3 -d clusters, surface diffusion may become ineffective in replenishing the loss due to thermal desorption. Then the average apparent thickness may stay low. Fortunately, as shown below, surface diffusion is always fast enough to replenish the desorption loss as long as the 3 -d clusters are not depleted.

As can be seen in Fig. 4(e), further heating above $930 \mathrm{~K}$ eventually caused the apparent thickness of the thick films to drop abruptly, all to about 1 TDML, and then the drops slow down. The temperature the abrupt drop occurs depends on the initial coverage of the film and is higher for thicker film. Also, the higher temperature the abrupt drop occurs, the larger the abruptness of that drop, which simply reflects the fact that the desorption rate increases significantly with temperature in this range. Since the drop is abrupt only for apparent thickness in between 1.9 and 1 TDML, it must be due to depletion of the respread film itself (which floats on top of the wetting layer). Below one TDML, the slowing down of the drop is consistent with a reduced thermal desorption rate observed for sub-monolayer coverage. For films with coverage below 1.8 TDML, the abrupt drops occur at about $930 \mathrm{~K}$. This means desorption of the re-spread film at $930 \mathrm{~K}$ is already very significant. However, for the thickest film shown, although desorption from the respread film must be no less significant, it does not cause decrease of the apparent thickness at $930 \mathrm{~K}$. Instead, the apparent thickness increases slightly as temperature increases and the abrupt drop is postponed until above $1000 \mathrm{~K}$. This is possible only if the desorption loss of the 2-d Ag gas is fully replenished by those invisible 3 -d clusters. It thus seems most plausible that the drops in apparent thickness occur only when the 3 -d clusters are exhausted.

Although Auger data alone cannot tell us for sure whether the 3-d clusters are exhausted when the abrupt drop in apparent thickness occurs,
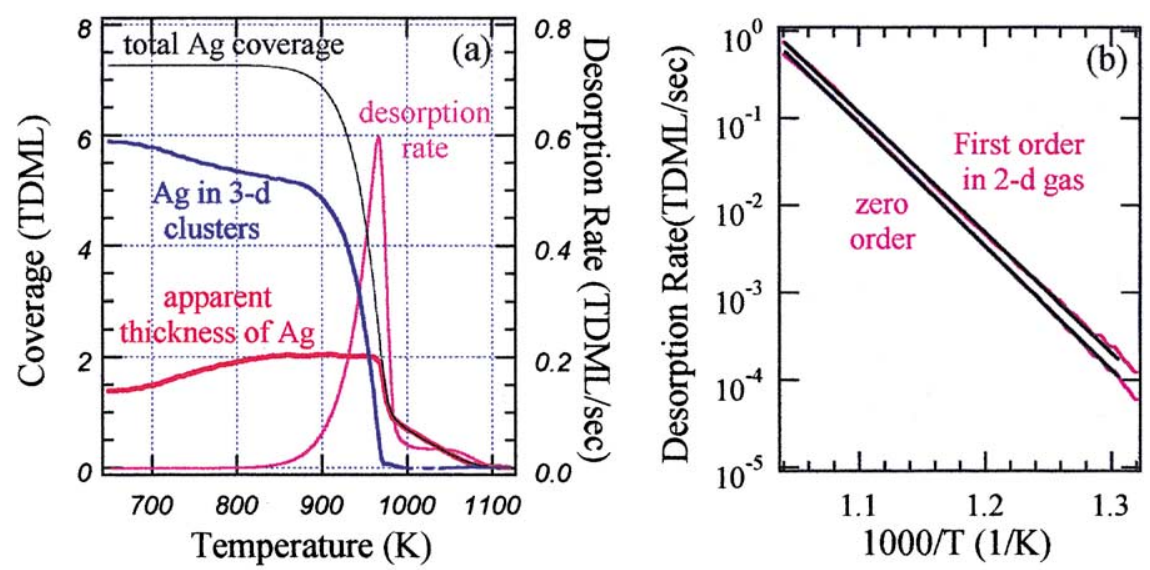

Fig. 5. Desorption of 7.2 TDML thick Ag films from Mo(1 11 1) as observed from Auger and mass analyzer. Sample heating rate is $4 \mathrm{~K} /$ s. (a) The purple curve is the measured desorption rate. By integrating the desorption rate backward, we obtain the black solid curve, which is the total amount of Ag remaining on the surface. The red curve is the apparent thickness of the film deduced from Auger. By subtracting the apparent thickness from the total amount of Ag, we obtain the blue curve, which is the amount of silver in the 3-d clusters. It drops to a very small value at $970 \mathrm{~K}$ when the abrupt drop of the apparent thickness begins. This supports the view that so long as the 3-d clusters still exist, they can always replenish the desorption loss of the 2-d gas. (b) Arrhenius plot of the leading edge (the part in between 760 and $960 \mathrm{~K}$ ) of the desorption rate assuming different order of kinetics. See text for more detail. 
together with thermal desorption data, they can. Fig. 5(a) shows results of experiments with two Ag films of the same coverage of 7.2 TDML. Both films had been deposited and annealed up to $750 \mathrm{~K}$ (at $4.0 \mathrm{~K} / \mathrm{s}$ ) and cooled down. Thus $3-\mathrm{d}$ clusters have formed. The two films are then heated at 4.0 $\mathrm{K} / \mathrm{s}$ rate up to $1300 \mathrm{~K}$. In one experiment, the apparent thickness is measured (red squares), while in the other, thermal desorption rate (purple curve) is measured. The thermal desorption rate is then integrated "backward" in time to produce the total amount of $\mathrm{Ag}$ left on the surface (black curve). The amount of Ag that hides inside the 3-d clusters is then obtained by subtracting the apparent thickness from the total amount of $\mathrm{Ag}$, which is shown as the blue curve. As can be seen, the amount of $\mathrm{Ag}$ in the 3-d clusters drops to zero when the drop in apparent thickness occurs. This confirms our view that as long as the 3 -d clusters exist they can always fully replenish the desorption loss of $\mathrm{Ag}$ in the re-spread film via surface diffusion. Thus, although thermal desorption may introduce some minor correction to the observed film thickness, the saturation is not an artifact due to desorption loss but an intrinsic surface property. That is, there is simply no tendency for $\mathrm{Ag}$ to spread into a film thicker than 2 TDML.

Since the thickness of the re-spread film is significant only at high temperature and $\mathrm{Ag}$ agglomerates back to 3-d clusters at low temperature, binding of $\mathrm{Ag}$ to the $\mathrm{Ag}$ wetted $\mathrm{Mo}\left(\begin{array}{lll}1 & 1 & 1\end{array}\right)$ surface must be weaker than binding to the 3-d Ag clusters. This should make desorption from the respread film easier than from the surface of a 3-d cluster. Also, the surface area of the re-spread film should be very much larger than the total surface area of all the 3-d clusters combined. Thus, when the thickness of the re-spread film is high, it seems likely that desorption should be dominantly from the re-spread film. More specifically, in between 800 and $960 \mathrm{~K}$ where the apparent thickness is almost constant, desorption should follow zero order kinetics. Is this true? Fig. 5(b) shows the Arrhenius plot of the leading edge region (760-960 $\mathrm{K})$ of the same desorption rate as shown in Fig. 5(a). As can be seen, it fits nicely with a straight line (shown in black). The best fit desorption activation energy is $2.80 \mathrm{eV}$. If we further assume the desorption rate to be proportional to the density of the re-spread film, then we can calculate the "desorption rate per coverage of the re-spread film", which is shown as the red curve. Again it fits nicely with a straight line (shown in black), but with slightly lower activation energy of $2.72 \mathrm{eV}$. Both activation energies are lower than the heat of sublimation from bulk Ag, which is $2.95 \mathrm{eV}$ [14]. Assuming there is no energy barrier for adsorption, then the above desorption activation energy is simply the binding energy of $\mathrm{Ag}$ in the re-spread film phase. Since binding energy of Ag to the 3-d clusters must be close to the bulk heat of sublimation, it should thus be about $0.15-0.23 \mathrm{eV}$ larger than the binding energy of $\mathrm{Ag}$ in the re-spread film, consistent with our assumption that it is small.

\subsection{Nucleation of the 3-d clusters}

For the thick films with initial coverage larger than 2.5 TDML, our observations indicate very fast response of the film to temperature changes. The apparent thickness of the system of 3-d clusters plus the re-spread film behaves in a rather predictable way. We now discuss films with initial coverage in between 1 and 2.5 TDML, which show much more complicated behavior. In the following, we discuss the results of annealing a series of Ag films, which are deposited with the sample temperature at or below $120 \mathrm{~K}$. The sample is then heated up to 850 at $1 \mathrm{~K} / \mathrm{s}$ (process $\mathrm{A}$ ) and then cooled down also at a rate of $1 \mathrm{~K} / \mathrm{s}$ (process $\mathrm{B}$ ). The sample is heated (process $C$ ) and then cooled (process D) again at the same rate and finally the sample is heated at a rate of $2 \mathrm{~K} / \mathrm{s}$ all way up to $1200 \mathrm{~K}$ (process E).

Fig. 6(a) and (b) shows the apparent thickness of this series of films during the first heating process (A) after the film is deposited. During this initial heating, we note that for all films with initial coverage above 1.1 TDML, they show a "primary" drop of apparent thickness. We have marked the midway point of the primary drop of the apparent thickness with a small circle. What caused these "primary" drops? First, these drops are not caused by thermal desorption as the temperature is too low. Examination at the edge of the 

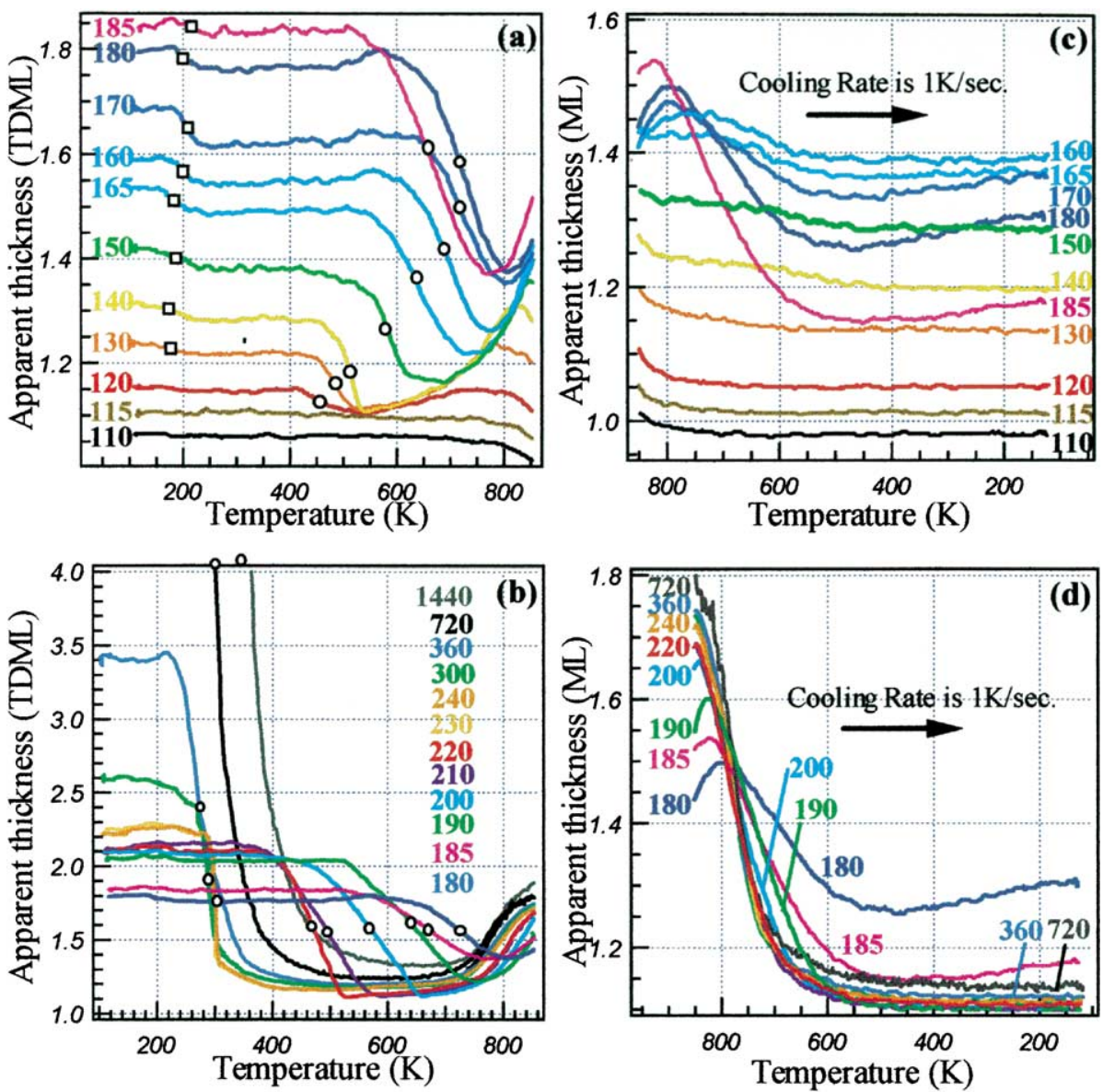

Fig. 6. Apparent thickness of a series of Ag films as a function of temperature when heated for the first time after deposition (process A, shown in (a) and (b)) and then cooled (process B, shown in (c) and (d)). Note the unusual way that temperature is plotted in (c) and (d). This is done in order for the implicit time arrow to always point to the right. Figure (a) and (c) are for films with initial coverage in between 1.05 and 1.85 TDML while figures (b) and (d) are for films with initial coverage in between 1.8 and 14.4 TDML. All films are deposited at or below $120 \mathrm{~K}$. Both heating and cooling rates are $1 \mathrm{~K} / \mathrm{s}$ and the maximum temperature reached is $850 \mathrm{~K}$. The number labeling each curve is deposition time in seconds. For some curves in (a), a square near $200 \mathrm{~K}$ marks the drop of apparent thickness due to phase separation into two 2-d phases with different thickness. For each curve in (a) and (b), there is a small circle indicating the midway point of the primary drop of the apparent thickness due to 3-d cluster formation. The 3-d clusters will re-spread into a 2-d gas at high temperature. Also, above $800 \mathrm{~K}$, thermal desorption caused some loss of $\mathrm{Ag}$. This is clearly reflected in reduction of apparent thickness for films with coverage lower than 1.5 TDML. When the films are cooled, if the coverage is larger than 2.5 TDML, the 2-d gas readily condenses back to existing 3-d clusters, and the resulting surface has an apparent thickness of 1.1 TDML below $600 \mathrm{~K}$. For films with initial coverage lower than 2 TDML, however, the 2-d gas do not necessarily condenses into 3-d clusters but can be incorporated into 2-d phases with apparent thickness up to 1.4 TDML.

back surface with Auger indicates the presence of Ag only after annealing at $700 \mathrm{~K}$ for $30 \mathrm{~min}$. Thus, diffusion to the back of the crystal is too small to account for the drop, which is rather uniform across the whole front surface. Is it diffusion of $\mathrm{Ag}$ into the bulk of Mo? Note, however, that (1) The bulk binary phase diagram indicates $\mathrm{Ag}$ and $\mathrm{Mo}$ are mutually insoluble. (2) It is hard to imagine why diffusion into the bulk is easier (i.e. occurs at lower temperature) and faster for the very thick films than the rather thin films (e.g. comparing the films with initial coverage of 14.4 and 1.3 TDML). 
(3) Our TPD result in Fig. 1 indicates Ag in excess of 1 TDML is bound in a state that can desorb more easily than those $\mathrm{Ag}$ of the first monolayer. All these together strongly indicate that although the deposited Ag become invisible by Auger, they are still on the surface. Thus, the only possibility is the well-known Stranski-Krastanov mode of thin film growth; i.e. formation of sparsely distributed "large" (as compared with the average film thickness) 3-d clusters on top of a wetting layer.

The cluster formation process showed a surprising dependence on the initial coverage. As shown in Fig. 6(a), the midway temperature is about $450 \mathrm{~K}$ for film with initial coverage of 1.15 TDML. This temperature increases quickly with initial coverage and at 1.8 TDML reaches a maximum of about $720 \mathrm{~K}$. Further increase of initial coverage caused it to drop quickly (cf. Fig. 6(b)), reaching a minimum of $270 \mathrm{~K}$ at 3.4 TDML. Afterward, the midway temperature increases again with coverage but only very slowly. Before the experiment, we speculated that for 3-d cluster formation, if the available $\mathrm{Ag}$ is so abundant as to form a thick continuous film, then the critical process is probably breaking up or cracking of the film. In this case, it may take more energy for thicker film to break up in the first place and after it breaks up, there is more material to be moved around in order for the apparent thickness to drop by one half. Both factors would lead to a higher midway temperature for thicker film. This seems in qualitative agreement with the observed behavior when the coverage is larger than 3.4 TDML. We also reasoned that, if there is not much available $\mathrm{Ag}$ around, then $\mathrm{Ag}$ atoms have to come together to form the 3-d clusters. Chances for the transient gathering to reach critical size should be strongly enhanced if there are more of them around. So the "midway temperature" for 3-d cluster formation should decrease when coverage increases. The large increase of the midway temperature in the coverage range from 1.1 to $1.8 \mathrm{TDML}$ is just opposite to our expectation. As shown below, closer examination of these films indicated that before forming 3-d clusters, Ag has already undergone phase separation into regions of $1 \times 6$ wetting layer and regions of $2 \times 12$ planar phase with higher coverage. Thus, gathering of enough Ag together seems never a problem. Instead, conversion from the planar ordered $2 \times 12$ structure to a $3-d$ cluster is the crucial step.

LEED pattern of samples annealed up to $350 \mathrm{~K}$ indicates formation of $1 \times 6(1.24$ up to $1.8 \mathrm{TDML}$ and 2.24 up to $9.0 \mathrm{TDML}$ ) and $2 \times 12$ (1.5 up to 2.05 TDML) structures. In particular, we note that for films with coverage in between 1.24 and 2.0 TDML, the LEED patterns seem to be linear combinations of the $1 \times 6$ and the $2 \times 12$ patterns. The pattern is purely $1 \times 6$ structure at the coverage of 1.24 TDML (Fig. 3(g)), and purely $2 \times 12$ structure at 2.05 TDML (Fig. 3(h)). Thus, although 3-d cluster formation has not yet occurred, the surface has already phase separated into areas of high coverage ( $~ 2$ TDML) $2 \times 12$ structure and low coverage $(\sim 1.2$ TDML) $1 \times 6$ wetting layer. Since the diffuse background of LEED is weak and shows no apparent coverage dependence in this coverage range, the domains are rather large. Temperature dependence of the LEED patterns indicates that the $2 \times 12$ structure is already well formed after being annealed at $250 \mathrm{~K}$, but not at $200 \mathrm{~K}$. Thus the phase separation should occur somewhere around $200 \mathrm{~K}$. Since the phase separation changes a uniform film into thinner and thicker region, the overall Ag Auger signal should drop slightly as part of the $\mathrm{Ag}$ is now buried deeper. It turned out that such drop were actually observable. As indicated by the squares in Fig. 6(a), for films with coverage from 1.2 to 1.85 TDML, there are small but clearly identifiable drops in apparent thickness near $200 \mathrm{~K}$. The magnitudes of such drops are shown in Fig. 7(b) as solid squares. The three solid curves shown are calculated differences between the apparent thickness of a statistically uniform film of coverage $\theta$ and that of a well phase separated surface. After phase separation, a fraction $\alpha(0 \leqslant \alpha \leqslant 1)$ of the surface area has coverage $\theta_{\mathrm{a}}$ and the rest of the surface has coverage $\theta_{\mathrm{b}}$. Once the pair of coverage $\left(\theta_{\mathrm{a}}, \theta_{\mathrm{b}}\right)$ is given, there is no adjustable parameter in calculating the apparent thickness using Eqs. (1)(3). As can be seen, although the uncertainty of the experimental data is not small enough to help decide $\left(\theta_{\mathrm{a}}, \theta_{\mathrm{b}}\right)$ to a better precision, the general agreement is strong support that there is indeed a 

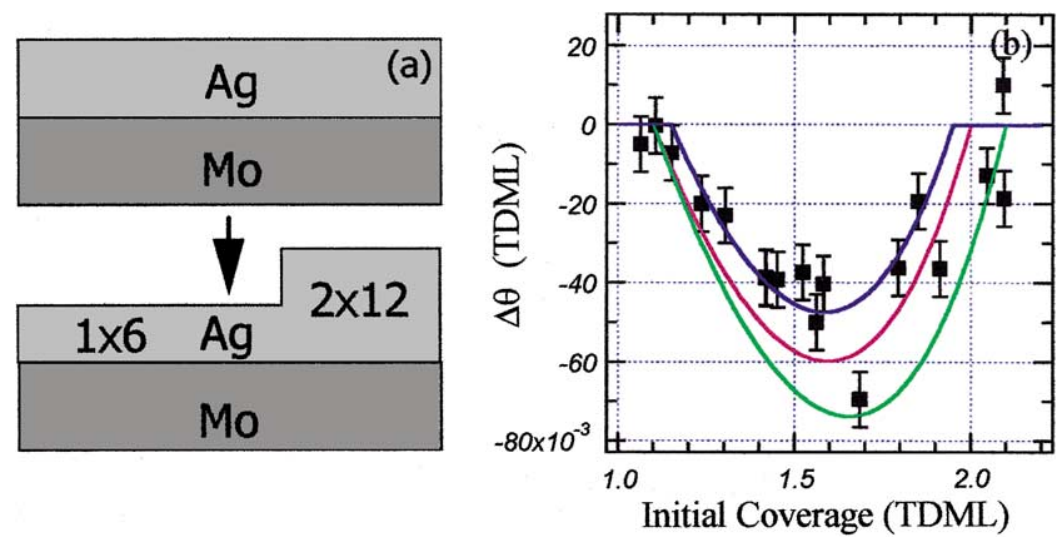

Fig. 7. Change of apparent thickness $(\Delta \theta)$ due to phase separation into two 2-d phases of different thickness. (a) shows the schematic of an initially uniform film phase separates into large domains of two different phases A (of coverage $\theta_{\mathrm{a}}$ ) and $\mathrm{B}$ (of coverage $\theta_{\mathrm{b}}$ ). The total area of phase $\mathrm{A}$ is of fraction $\alpha$ of the total surface area, while the total area of phase B is of fraction $(1-\alpha)$ of the total surface area. $\theta=\alpha^{*} \theta_{\mathrm{a}}+(1-\alpha)^{*} \theta_{\mathrm{b}}, 0 \leqslant \alpha \leqslant 1$ (b) shows $\Delta \theta$, change of apparent thickness due to phase separation. The solid squares are experimental data. The apparent thickness $\theta^{\prime}$ of the phase separated surface can be calculated using Eqs. (1)-(3). The curves are calculated $\Delta \theta$. Once $\left(\theta_{\mathrm{a}}, \theta_{\mathrm{b}}\right)$ is given, there is no adjustable parameter. $\Delta \theta$ as a function of $\theta$ for three sets of $\left(\theta_{\mathrm{a}}, \theta_{\mathrm{b}}\right)$ are shown: the upper (blue) curve is for $(1.15,1.95)$, the central (red) curve is for $(1.1,2.0)$ and the lower (green) curve for $(1.1,2.1)$. Note that the amplitude of the calculated $\Delta \theta$ is a strong function of the difference between $\theta_{\mathrm{b}}$ and $\theta_{\mathrm{a}}$ but rather insensitive to an overall shift.
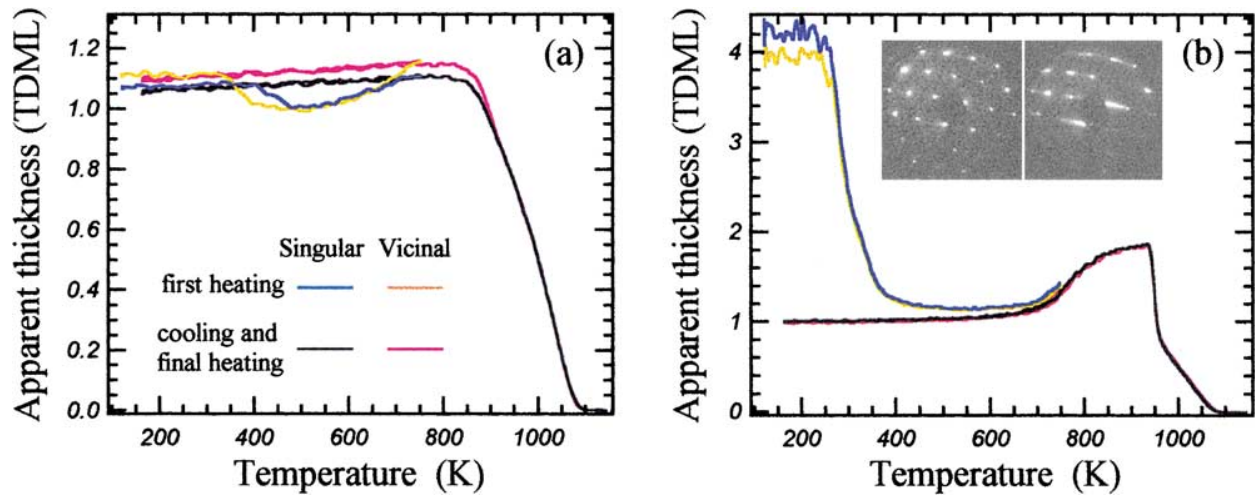

Fig. 8. Influence of steps on the formation of 3-d clusters and density of the re-spread film. See text for specification of the vicinal surface. For both experiments shown in (a) and (b), Ag is deposited at $120 \mathrm{~K}$, the sample is then heated up to $750 \mathrm{~K}$ at $4 \mathrm{~K} / \mathrm{s}$. Data obtained during this initial heating is shown in blue (singular surface) or orange (vicinal surface). The surface is then cooled at a rate of as close to $4 \mathrm{~K} / \mathrm{s}$ as possible down to about $150 \mathrm{~K}$ and heated up again at $4 \mathrm{~K} / \mathrm{s}$ all way to $1300 \mathrm{~K}$. Data for the cooling and final heating part is shown in black (singular surface) or red (vicinal surface). LEED patterns of the singular and the vicinal clean surface are shown as inset in (b). See text for explanation.

phase separation into thicker and thinner 2-d phases at about $200 \mathrm{~K}$.

A natural assumption is that 3 -d clusters would form from the $2 \times 12$ structure. This is true for films with coverage below 1.4 TDML. For these films, as the 3-d cluster formation occurred below
$500 \mathrm{~K}$, it should only involve the transformation of high coverage planar $2 \times 12$ phase into 3 -d clusters. For films labeled 160, 170 and 180, the apparent thickness increases slightly in between 500 and $550 \mathrm{~K}$. This should mean the phase-separated surface is resuming a more uniform distribution of 
Ag again. Indeed, for these surfaces, both $1 \times 6$ and $2 \times 12$ superlattice spots in the LEED pattern disappeared after being annealed at $550 \mathrm{~K}$ and the LEED pattern now looks like that shown in Fig. $3(\mathrm{k})$ which is characterized by a ring around the specular spot. Thus, for these coverage, there seems a structural change at $550 \mathrm{~K}$ to certain planar "ring phase" before the formation of 3-d clusters. For even thicker films, the 3-d cluster formation temperature dropped with increasing coverage and for initial coverage larger than 2.3 TDML, the midway temperature of 3-d cluster formation has dropped to about $300 \mathrm{~K}$. For these films, once 3-d clusters are formed, the $2-\mathrm{d}$ films in coexistence with the $3-\mathrm{d}$ cluster also have the same $1 \times 6$ structure (so long as the annealing temperature has never been raised above $500 \mathrm{~K}$ ). Thus, at temperatures lower than $500 \mathrm{~K}$, the stable wetting layer assumes the $1 \times 6$ structure and before $3-\mathrm{d}$ cluster formation, Ag already aggregates together, forming large 2-d islands of $2 \times 12$ phase (about 2 TDML thick) or films thicker than two monolayers.

\subsection{Influence of steps on 3-d cluster formation}

To get some feeling of the role steps play in the formation of 3-d clusters and its influence to the re-spread film, we have taken advantage of a sample whose surface has become rounded during electropolishing. Fig. 8 shows data obtained from two regions of the same crystal, one region is singular, while the other is vicinal to (111). From LEED, the vicinal region has a miscut angle of $3.5^{\circ}$, with steps running along $[1,-1,0]$ direction and a high step density of $0.65 / \mathrm{nm}$. As can be seen from Fig. 8(a), for film with coverage slightly larger than the wetting coverage, the starting temperature of 3-d nucleation on the vicinal surface is lower than that of the singular surface by a large amount of $55 \mathrm{~K}$. This indicates a strongly enhancing effect of steps to the 3-d nucleation process at low coverage. However, for temperature in between 500 and $700 \mathrm{~K}$ where the 3-d clusters evaporate into a 2-d gas, the density of the 2-d gas shows no difference for the two surfaces. For films about 4 TDML thick, the result is shown in Fig. 8(b). In this case, neither formation of 3-d cluster nor the density of the 2-d gas is influenced by the existence of steps.

For the 3-d cluster formation process, if cracking of the thick film is the crucial step, lack of influence by the steps seems not surprising. For the 2-d gas, lack of any influence on its density seems to indicate that the step sites are not much more attractive than the terrace sites. This is probably a distinctive characteristic for steps on an atomically rough surface, which is not hard to conceive once we examine a hard sphere model of the (1 111$)$ surface as shown in Fig. 9. In the case of a close packed surface such as Mo(1 10$)$, we would expect $\mathrm{Ag}$ to bind more strongly to the steps and diffuse more rapidly along the steps. Thus, if a 3-d cluster start to dissociate on a close packed surface, it is likely that the dissociated $\mathrm{Ag}$ will preferentially travel along the steps and populate alongside these "highways" consisting of steps. If this is true, then there should be a temperature range when the 2-d population is proportional to the density of the steps. Since we do not see any dependence on step density, it suggests that step sites on a rough surface is not as attractive as step sites on a smooth surface. Nevertheless, when the density of steps is high, it should be a big disturbance to large superstructures such as the $2 \times 12$ (a unit cell is shown on Fig. 9), and such disturbance may very well raise the energy of the 2-d film and make them more unstable.

\subsection{The ring phase}

There is another interesting aspect in Fig. 8(a). As shown by either the red (vicinal surface) or the black (singular surface) curve, once the sample has been annealed up to $750 \mathrm{~K}$, reduction of apparent thickness due to 3-d cluster formation no longer occurs! This is true either when the sample is being cooled down or when the sample is later being heated up the second time. That is, on both the singular and the vicinal part of the surface, annealing up to $750 \mathrm{~K}$ somehow caused the resulting 2-d films to become more stable! As stated previously, LEED of samples $(\theta>0.3)$ annealed above $550 \mathrm{~K}$ all show patterns similar to the one shown in Fig. 3(k). Thus, in addition to $1 \times 6$, we have another class of 2-d "ring phase" that's even more 


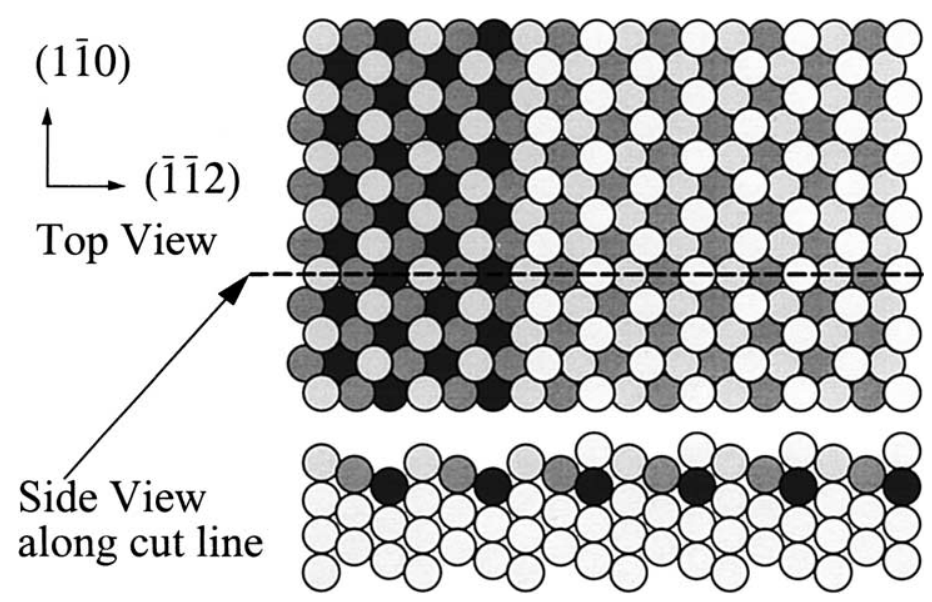

Fig. 9. Hard sphere model of (1 111$)$ surface of body centered cubic crystal structure. Also shown are cross sectional view of a step structure along $[1,-1,0]$. Such model strongly suggests that steps on an atomically rough surface such as bcc(1 1 1) is qualitatively different from steps on a close packed smooth surface. Due to the rough nature of the (1 111 ) surface, difference (say number of nearest neighbors) between terrace site and step edge site is much less prominent than that on a close packed surface. Very likely, diffusion along the steps is not much different from diffusion on the terrace.

stable but accessible only after high temperature anneal. Since qualitatively similar LEED patterns are obtained after high temperature annealing of films with coverage above 0.3 TDML, the "ring phase" can accommodate a very large range of coverage indeed! Although the "ring phase" is apparently stable at a coverage slightly above the wetting coverage, Figs. 6(d) and 8(b) indicates that when there are 3 -d clusters around, the "ring phase" in equilibrium with the 3-d clusters can only have the wetting coverage. Thus, the apparent stability of films in Fig. 8(a) should partly be due to thermodynamics and partly be due to kinetics. That is, we believe in principle its energy could be further lowered by forming large but very sparsely distributed 3-d clusters on top of a film of just the wetting coverage. Annealing at high temperature, however, caused the available Ag to re-spread uniformly across the surface and readily forms the "ring phase". Since the "ring phases" are more stable than a lot of small 3-d clusters on top of a film of the wetting coverage, nucleation of new 3-d clusters cannot occur and the film is kinetically trapped in the "ring phase".

How thick can these "ring phase" be? An examination of Fig. 6(c) would suggest that the coverage of the ring phase formed gradually in- creases with initial coverage until it reach a maximum of 1.4 TDML for an initial coverage of 1.6 TDML. As mentioned in the introduction, authors of Ref. [3] observed re-spreading of Ag 3-d clusters after the sample is cooled down. We believe the spreading they observed is due to this high-density ring phase instead of the high temperature respread film. For initial coverage above 1.6 TDML, we note two peculiar behaviors of those curves labeled 170, 180 and 185 in Fig. 6(c). First, as temperature start to drop, the apparent thickness do not reduce, but kept on increasing. We will address this point later. Then the apparent thickness dropped as usual, indicating formation of 3-d clusters. However, when the temperature falls below about $450 \mathrm{~K}$, the apparent thickness increased again! This seems to indicate that the competition between the ring phase and the 3-d clusters are so subtly balanced that a drop in temperature can cause Ag to flow from the 3-d cluster phase to the ring phase. Note in particular that once Ag is removed from the 3-d clusters, the clusters will become smaller and presumably become less stable. This may be one of the reasons why this strange spreading during cooling can proceed even at very low temperature. If the initial coverage is above 2 TDML, spreading of 3-d clusters during cooling 
no longer occurs. This should be due to increased size of the 3-d clusters, which make the 3 -d clusters energetically the more favored phase.

\subsection{Density and size of the 3-d clusters}

The complete annealing history for some of the films with initial coverage larger than 2 TDML are shown in Fig. 10. These are from the same set of experiments, which has been partly shown in Fig. 6. However, to emphasize the hysteresis in between 600 and $850 \mathrm{~K}$, only data above $400 \mathrm{~K}$ are included. Obviously, for the thickest film as shown in Fig. 10(a), once the 3-d clusters formed, the apparent thickness depends mainly on temperature and is almost the same whether the sample is being heated or cooled. In contrast, in Fig. 10(f), the apparent thickness of the surface when being cooled (blue and green curves) is significantly larger than that of the surface when being heated (red, orange and black curves). That is, for some of the films, the apparent thickness shows hysteresis during temperature cycling,

What caused the hysteresis? If for some reason it takes a very long time for the re-spread film to either fill the surface or agglomerate back to the $3-\mathrm{d}$ clusters, then the heating and cooling rate of $1 \mathrm{~K} / \mathrm{s}$ may simply be too fast for some of the films to remain in equilibrium and thus show hysteresis. There are evidences supporting this view.

Note in Fig. 6(c) and (d), for films with labels ranging from 160 to 200 (corresponding to initial coverage in between 1.6 and 2.0 TDML), the apparent thickness did not decrease immediately upon cooling but kept on increasing for a while. Closer examination reveals that for the increasing part of these curves, the apparent thickness is always below the apparent thickness attained by thicker films at the same temperature. For example, in Fig. 6(d), the curves labeled 180, 185, 190 and 200 all kept on increasing even after cooling starts. But such increase stops when the curves nearly hit the red curve (labeled 220) from below, irrespective of the actual temperature this happens. (Since the apparent thickness of the red curve labeled 220 reduces immediately upon cooling, the apparent thickness of $\mathrm{Ag}$ cannot be too far away from equilibrium density and thus can serve as a good comparison standard.) These behaviors strongly suggest that for these films, somehow spreading of Ag all over the surface is slow, and the apparent thickness is falling far below the equilibrium thickness as determined by the raising temperature. When cooling starts, the apparent thickness of $\mathrm{Ag}$ is still far below the equilibrium value and thus $\mathrm{Ag}$ kept on spreading. As the temperature dropped further, the re-spread $\mathrm{Ag}$ film eventually become supersaturated and then Ag starts to agglomerate back to the 3-d clusters. A good indicator of the slowness of the respreading is the apparent thickness attained when the sample is at the highest annealing temperature of $850 \mathrm{~K}$. It is thus no accident that in Fig. 6(d), only films with a low apparent thickness at $850 \mathrm{~K}$ "overshoots" when cooling starts. Moreover, just as spreading is slow, agglomeration should also be retarded. Indeed, as shown in Fig. 6(b) and (d), the smaller the apparent thickness attained at $850 \mathrm{~K}$, the slower the apparent thickness reduces during agglomeration. Thus, the observed hysteresis is due to the slowness of the film's response in both re-spreading and agglomeration. Dissociation from (or association to) the cluster and surface diffusion are the two major activities involved in re-spreading (or agglomeration). Why should these activities be more effective for some films (e.g. Fig. 10(a) and (b)) while less effective for other films (e.g. Fig. $10(\mathrm{e})$ and (f))?

To our belief, the key factor is the number density of the 3-d clusters for any particular film of interest. Obviously, for films with a lower density of the 3-d clusters, each cluster has to spread to a larger area in order for the whole surface to be covered. Spreading to a larger area definitely will take longer time. When cooling, all Ag atoms have to find their way to a certain 3-d cluster. When there are less such clusters nearby, it also takes longer for the $\mathrm{Ag}$ atoms to find one. Simple dimensional argument would require both spreading and agglomeration times to be proportional to $(n D)^{-1}$, where $D$ is the surface diffusion constant and $n$ the number density of the clusters. Thus, the smaller the density of the 3-d clusters, the more time it will take for surface diffusion to bring the surface to approach new equilibrium as temperature changes. Moreover, since either dissociation 


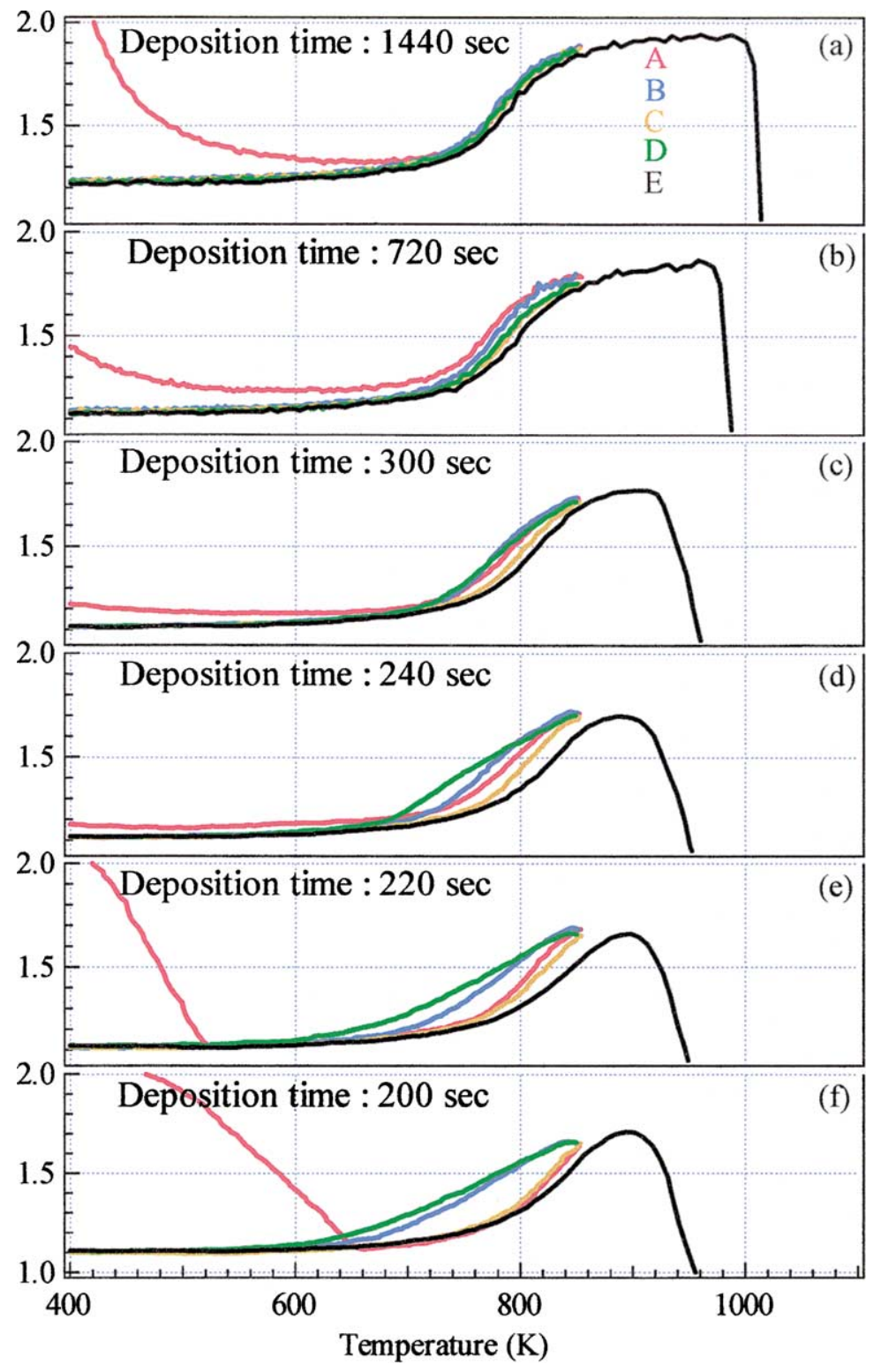

Fig. 10. Apparent thickness of samples with various initial coverage $\theta$ as a function of temperature when subject to three heating and cooling cycles. Red curve is for initial heating process A with $1 \mathrm{~K} / \mathrm{s}$ rate up to $850 \mathrm{~K}$. Blue curve is for cooling process $\mathrm{B}(-1 \mathrm{~K} / \mathrm{s})$ which follows A. Orange curve is for heating process C $(1 \mathrm{~K} / \mathrm{s})$ which follows B. Green curve is for cooling process D $(-1 \mathrm{~K} / \mathrm{s})$ which follows C. The final process E (black curve) is heating at $2 \mathrm{~K} / \mathrm{s}$ all way up to $1200 \mathrm{~K}$, shown in black. As shown, for $\theta>2 \mathrm{TDML}$, the higher the coverage is, the smaller the hysteresis in apparent thickness when temperature is cycled. This should mainly be due to a higher density of 3 -d clusters.

from or association to the clusters has to happen at the boundary of the clusters, the total rate should be proportional to the sum of the perime- ters of all the clusters. Due to mass conservation, the total length of perimeters per unit area is proportional to $\theta^{1 / 3} n^{2 / 3}$, where $\theta$ is the average 
coverage and $n$ is the number density of clusters. Thus, small number density of clusters could not only reduce the effectiveness of surface diffusion, it could also reduce the total rate of dissociation from (or association to) the clusters. The data in Fig. 10 certainly suggests very strongly that for films with initial coverage larger than 1.8 TDML, the larger the initial coverage of a film, the higher is the density of the 3-d clusters formed. There are independent evidences supporting this proposition. Note that if the density of the 3-d clusters is so high that their contribution to the Auger signal can no longer be neglected, then the observed apparent thickness will increase. In Fig. 10(a), note that after two annealing cycles and cooled down to low temperature, the apparent thickness of this initially thickest film does not reduce to 1.1 TDML but to a value slightly higher than 1.2 TDML. To a lesser degree, similar increase can be found for the curves labeled 720 and 360 in Fig. 6(d). Such apparent increase of the thickness of the wetting layer is indicative of a very high density of 3-d clusters. From all these evidences, we are convinced that much more dense population of 3-d clusters exist on films with higher initial coverage.

Why should the thicker film have a more dense population of 3-d clusters? An examination of Fig. 6(b) indicates that the cluster formation temperature drops drastically from about 720 to $270 \mathrm{~K}$ for films with initial thickness ranging from 1.8 to 3.6 TDML. This suggests a strong correlation between the formation temperature and the number density of the clusters formed. Our speculation is that the size of the newly formed 3-d cluster is limited by the range it can collect $\mathrm{Ag}$. Without long-range interaction and competing clusters, the diffusion length of a typical Ag atom determines this collecting range. Thus, if nucleation occurs at low temperature, due to small diffusion length, the clusters formed should be small and the density should be high. Conversely, if the nucleation occurs at high temperature, then the clusters formed will be large but the density of the clusters will be low.

Is there anyway to tell the relative importance of surface diffusion and dissociation/association in the spreading/agglomeration process? Fig. 11 shows results of an experiment in which films de-

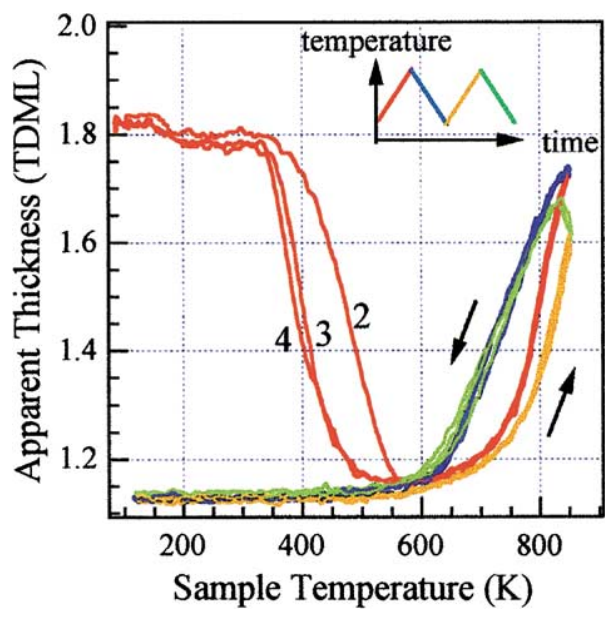

Fig. 11. An experiment to study the cluster formation and respreading of films grown on pre-seeded surface. 2.1 TDML of $\mathrm{Ag}$ is first deposited at $110 \mathrm{~K}$ on a clean surface and subject to two heating and cooling cycles of $1 \mathrm{~K} / \mathrm{s}$ rate to create the seeded surface. Then 0.7 TDML of $\mathrm{Ag}$ is deposited on top of the seeded surface at $110 \mathrm{~K}$ and the sample is again subject to two cycles of heating and cooling. This process of deposition followed by two cycles of heating and cooling is repeated twice more. The apparent thickness of the three seeded films during all the heating and cooling cycles is plotted. The first heating process after each deposition is shown in red, the following cooling process shown in blue, the second heating process shown in orange and the second cooling process shown in green. Major feature is that as more materials are added, the hysteresis loop remains essentially the same, strongly suggesting that size of the 3-d clusters are not an important factor in determining the width of hysteresis. See text for details.

posited on a seeded surface are monitored. A clean surface is first deposited with 2.1 TDML of Ag (same as that for Fig. 10(f)) and subject to two heating/cooling cycles $(1 \mathrm{~K} / \mathrm{s}$ rate and a maximum temperature of $800 \mathrm{~K}$ ). The 3 -d clusters formed at this stage will serve as seed. We add 0.7 TDML to the surface at $120 \mathrm{~K}$ and again subject the surface to the same heating/cooling cycles twice while recording its apparent thickness. The whole process of deposition plus two annealing cycles is repeated two more times. The apparent thickness during all the heating/cooling cycles after the second, third and fourth deposition is shown in Fig. 11. There are differences in the initial annealing stage when the newly added film either forms new 3-d clusters or agglomerates into existing ones. However, the most drastic feature is that the re-spreading 
behaviors of these surfaces are essentially identical. Due to thermal desorption at high temperature, each heating/cooling cycle will cause a loss of about 0.15 TDML of Ag. Each deposition adds 0.7 TDML of Ag to the surface. Thus, after two heating/cooling cycles and one deposition, there are 0.4 TDML more Ag on the surface which gets into the 3-d clusters. All clusters should become bigger. However, no appreciable change in the behavior of apparent thickness can be observed. This is a strong evidence supporting the view that dissociation/association is not a rate limiting step in determining the width of the hysteresis as shown in Fig. 10. More likely, the distribution of the clusters are so sparse that hysteresis is mainly due to limited surface diffusion.

Another important feature in Fig. 11 is that for all three films, the observed apparent thickness during the second heating process is always smaller than that observed during the first heating process. Let us compare the second heating process after deposition 2 with the first heating process after deposition 3 . If the newly deposited material only diffuse to existing old 3-d clusters, then all clusters simply grow larger and the distribution of the clusters remains invariant. If this is the case, when the sample is heated above $600 \mathrm{~K}$, we expect its apparent thickness to behave in the same way as in the heating process before deposition. In reality, the apparent thickness is significantly larger. This can happen only if new clusters have formed. This will cause the number density of the clusters to increase. In addition, we believe the new clusters are also significantly smaller than the old ones.

From Fig. 11, new cluster formation after deposition 3 occurs at about $400 \mathrm{~K}$. Note that the old clusters have been collecting Ag from the re-spread film during the last cooling process. Since such collection is done above $650 \mathrm{~K}$, the collection range must be much larger than that at $400 \mathrm{~K}$ and thus the old clusters should be significantly larger than the newly formed ones. (If there are many old clusters in the same collecting range, then this argument seems flawed. However, the fact that new clusters can form at all indicates that even if there are competitors, each still has a territory larger than the collection range at $400 \mathrm{~K}$.) When the temperature is raised again, due to the small size and high density of the new clusters, the average apparent thickness increased faster. Actually, it seems that the new clusters are so small that one heating/cooling cycle is enough to make these newly formed small clusters re-spread and get collected by the old big clusters completely. Thus, in the second heating cycle, it is again the same old (but now even bigger) clusters that are playing the game. It should thus be no accident that for all the second heating processes, the apparent thickness behaves in the same way.

To summarize, the high density of the re-spread film makes the $\mathrm{Ag} / \mathrm{Mo}(111)$ a unique system for the study of nucleation, growth and ripening phenomena. Self-consistent propositions of what is happening on the surface can often be constructed from clues provided by temperature programmed Auger (TPA) data. However, additional information from in situ real space imaging techniques such as scanning electron microscopy [26] or scanning tunneling microscopy would be most desirable. If these can be combined with TPA in the future, then all pertinent information can be measured directly for construction of a more complete understanding.

\section{Discussion}

\subsection{Nature of the re-spread film}

What is the nature of the re-spread Ag after steady state is reached at a particular temperature? The LEED pattern of the sample at or above 600 $\mathrm{K}$ shows a bright uniform blur with strongly attenuated $1 \times 1$ spots (compared with that of the clean surface). This indicates a very disordered film. As the apparent thickness is continuously variable, the most plausible and straightforward answer is that the re-spread Ag forms a homogeneous 2-d gas-like [15-17] phase that floats on top of the wetting layer and fills up all the area not occupied by the 3-d clusters. By "gas", it is meant to imply that it has a natural tendency to expand and fill up the empty area not pre-occupied by $3-d$ clusters. In contrast, although a condensed liquid is also in a disordered state, normally [18] it will 
not automatically expand to fill up the space. Since there is a wetted substrate exerting a strong influence, particles of this 2-d gas are definitely not "moving freely" and the probability of finding anyone of them need not be homogeneous microscopically.

But how do we know the 3-d clusters do not spread into a 2-d ordered phase or a 2-d liquid? After all, above $900 \mathrm{~K}$, the density of the 2-d phase does reach 0.8 TDML and particles in that 2-d phase will stay in touch with each other. The answer is that liquid and ordered phases are incompressible and have a rather well defined density. If such a $2-d$ phase fills the whole area between $3-d$ clusters, the apparent thickness should have a welldefined value instead of being continuously variable over a large range. One could still argue that the spread Ag forms discrete 2-d patches with the total area occupied by the patches varying with the sample temperature. Then the observable average density can vary continuously with temperature. To rule that out, note the apparent thickness not only increases when temperature increases but also reduces reversibly when temperature is lowered, thus the spreading is not diffusion limited. One has to ask, then, what stops the 3-d clusters from spreading all the way until the $2-d$ condensed phase fills up all the area? After all, once a 3-d cluster spreads, it will become smaller and making it even easier to spread further.

One situation for which a 3-d cluster can stop spreading before filling up the whole area is when the 3-d cluster exhausts itself. Suppose the 3-d clusters have a wide distribution of sizes, and suppose that at lower temperature only smaller 3-d clusters spread while the larger clusters do not spread at all until the temperature is higher. As temperature increases, we will then observe a gradual increase of apparent thickness as larger and larger 3-d clusters spread. The trouble with this argument is that the spread Ag should readily be absorbed into nearby large surviving clusters when cooling down. The small 3-d clusters will disappear and only large ones are left. When the surface is heated again, we expect the apparent thickness to follow very different path. In particular, since the small 3-d cluster disappeared, rising of the apparent thickness will not occur until the big clusters spread. This should result in a sharp rise of apparent thickness at higher temperature. However, the experiment for thick films shows that the apparent thickness of Ag reproducibly follow the same slowly varying, "beaten" path when temperature is cycled. Thus, spreading of 3-d clusters at size dependent temperature into $2-\mathrm{d}$ condensed patches is not completely consistent with the experiment.

Finally, one could still argue that if some kind of inhomogeneity makes part of the surface more attractive than other part of the surface, then $\mathrm{Ag}$ will spread to the more attractive place at lower temperature and to the less attractive place at higher temperature. Step edges seems the only and the most likely candidates for creating such attractive potential. If this were the case, we would expect the rate of increase of apparent thickness to be proportional to the step density. More specifically, the rate of increase in apparent thickness should be much more prominent for vicinal surfaces which has much higher step density than the singular surface. Some experimental results of the effect of step edges are shown in Fig. 8(b). What is plotted are the apparent thickness as a function of temperature for two Ag films about 4 TDML thick, one is obtained from the singular part of the surface while the other obtained from the vicinal part of the surface. The vicinal part of the surface has steps aligned along [ $\left[\begin{array}{lll}1 & 1 & 0\end{array}\right]$ direction and a very high step density of $0.65 \mathrm{~nm}^{-1}$. Yet, other than the small difference in initial coverage, there is no observable difference in the behavior of apparent thickness from that of the singular surface. Although steps along another direction may behave differently, this result strongly suggests that steps do not play an important role in causing the increase of apparent thickness at high temperature. Since almost all arguments for spreading into a 2-d condensed phase leads to inconsistency with some experimental results, we are confident that the 3-d clusters spread into a 2-d gas like phase. For simplicity, we will use "2-d gas" instead of the correct "2-d gas-like phase" in the rest of the paper.

Why does the 2-d gas reach such high density? We postulate it is due to (1) a rather small difference in binding energy in favor of the 3 -d clusters 
instead of the 2-d gas, and (2) the 2-d gas has a larger entropy than the $3-\mathrm{d}$ clusters. Note that, for metals, the thermal excursion of surface atoms perpendicular to the surface is generally $50-100 \%$ greater than a bulk atom at the same temperature [19]. Also, the LEED observation of many large but soft super-lattice structures is compatible with large entropy associated with the $2-d$ phases. The free energy of the system is $E-T S$, where $T$ is the absolute temperature, $S$ is the entropy and $E$ the energy of the system. At low temperature, the energy term dominates and the 3-d cluster is the favored phase. As temperature increases, the entropy term gradually takes over and the balance will shift away from the 3-d cluster toward the 2-d gas which has larger entropy. Before the onset of thermal desorption, $\mathrm{Ag}$ has nowhere other than the 3-d cluster phase or the 2-d gas phase to go. Thus the system is a closed one similar to that of a pressure cooker. As the temperature is very high, it is not surprising that the density of the 2-d gas can build up to such unusual level.

\subsection{Nucleation of 3-d clusters}

Why are the planar phases most stable against 3 -d cluster formation when the Ag coverage is about 1.8 TDML? Some speculations follow.

(a) Independent islands: Since all our samples are annealed in the same way, it should be safe to assume that when reaching the same temperature, the sample with larger initial coverage will have larger 2-d islands. For 2-d islands of finite size, the average binding energy per atom should increase slightly with the size of the island as the relative abundance of the edge atoms is reduced. This could make the larger 2 -d islands more stable than the smaller ones. So it seems possible that due to a very small energy difference between the 2 -d island and the 3-d clusters, ready formation of larger 2-d island is the reason why films of higher coverage have higher stability against 3-d cluster formation. However, if this is the only mechanism, then maximum stability should be observed at the coverage of 2.05 TDML when the whole surface assumes $2 \times 12$ structure. That is, when the 2 -d island is infinitely large. This is not consistent with the experimentally observed $1.8 \mathrm{TDML}$. If we re- fine the above argument by comparing the average binding energies of a finite amount of film material in either 2-d island or 3-d cluster form, then it is possible that 2-d island of a particular size is most stable. However, without knowing the important parameters such as edge-energy of the 2-d island and surface energy of the 3-d clusters, no concrete prediction can be made for comparison with the experiment.

(b) In the argument above, we have considered the stability of the 2-d islands from an "independent island" point of view. If we compare atomic diameters based on hard-sphere models for Ag and Mo, we find the diameter of $\mathrm{Ag}$ to be 6\% larger than that of Mo. Thus, there could be a significant strain in a commensurate 2-d Ag film. The longrange interaction between the strain field of the 2-d islands may thus be important. It has been suggested [20] that for systems with planar domains, the long-range interaction among strain field induced by the domain walls will impose an optimum configuration of the 2-d domains. In particular, one model calculation [21] predicted that when the area fraction of one of the phases is 0.5 , stabilization due to isotropic strain interaction is maximized. Qualitatively, existence of a minimum energy state at certain coverage agrees with our experiment. However, experimentally the maximum stability occurs when the area fraction equals $(1.8-1.2) /(2.05-1.2)=0.7$. This seems not in good accord with the experiment. However, closer examination of Fig. 6(a) indicates that there are small rises of apparent thickness right before the 3 -d cluster formation for curves labeled 160, 170 and 180 . We believe these rise are due to conversion into a single phase which we called the "ring phase" (to be discussed later). Thus, it is possible that as coverage increases, strain interaction caused extra stability of the film and existence of the ring phase further extended the range of maximum stability.

(c) Once the initial coverage is above 2 TDML, the 3-d cluster formation temperature lowered very quickly. This is consistent with the fact that the 2-d gas saturates at about 1.9 TDML and our argument that once the coverage is above 2 TDML, it is much more favorable to form 3 -d clusters than to form a 2 -d film. This seems to 
suggest that the crucial step determining the stability of a 2-d island is the "climbing" of some atoms onto the third TDML. Once enough atoms successfully climbed on, the rest can follow and 3 -d cluster formation may then proceeds easily. If climbing up is the key issue, then the question is why climbing up should be most difficult at 1.8 TDML?

Of the above three speculations, only the mechanism involving strain interaction among domains provide some definite prediction, which is almost but not quite in accord with the experiment. Further theoretical calculation and study using STM to get real space image of the surface should help resolve some of the above mentioned possibilities.

\subsection{More on "ring phase"}

Rings in diffraction patterns have been reported before and it has been pointed out [22] that diffraction rings could be caused by randomly distributed identical islands or islands of varying diameter but fairly constant separations. In the case of $\operatorname{Ag} / \operatorname{Pt}\left(\begin{array}{lll}1 & 1\end{array}\right)$, it has been reported [23,24] that below $550 \mathrm{~K}$, large-scale metastable uniformly strained 2-d Ag islands grow pseudomorphically. Once annealed above $620 \mathrm{~K}$, Ag clusters of about $10 \AA$ size dissolved [24] in the top Pt layer $(\theta<0.5)$ or Pt clusters of about $10 \AA$ size dissolved in the Ag layer $(0.5<\theta<1.0)$. And there is evidence indicating such monolayer confined mutually dissolving form is the thermodynamically stable form. In our case of $\mathrm{Ag} / \mathrm{Mo}(111)$, we note that annealing below $550 \mathrm{~K}$ produces various super-structures while once annealed at or above 550 $\mathrm{K}$ into the ring phase, annealing at lower temperature for prolonged period of time has never brought back any of the superstructure spots. Thus, the ring phase should be thermodynamically more stable than any of the super-structures. If the clean surface of Mo has tensile stress, then as Ag has a larger atomic radius, it seems possible that mixing of small Ag 2-d clusters into a Mo layer can reduce the energy by relieving the tensile stress. Since Ag and Mo are mutually immiscible [25]

(a)

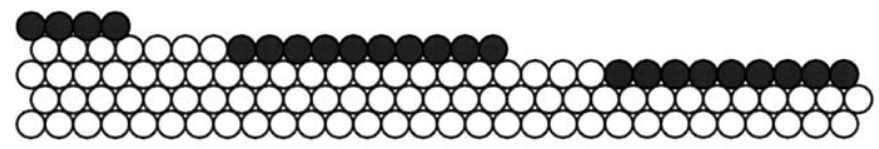

(b)

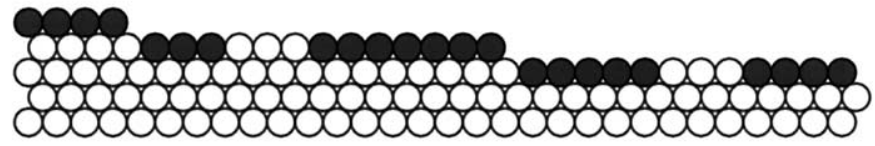

(c)

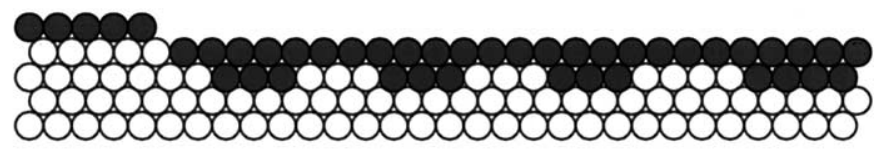

(d)

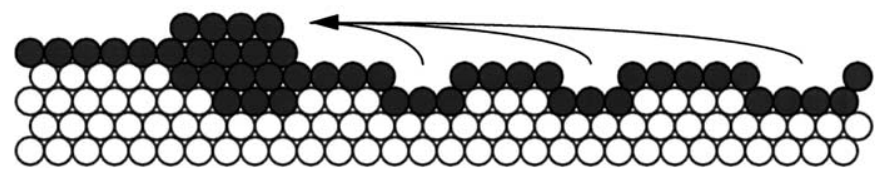

Fig. 12. Schematics showing various atomic arrangements where the film material is always on top or by the side of the substrate material. (a) Films grown near a step, no mixing with substrate. (b) Film material forms 2-d clusters, which mixed into top substrate ledges. For a film with coverage larger than one monolayer, ordinarily film materials will pile up on the surface (kinetically more accessible). However, it seems possible that the "excess" material could also dig into the substrate, as shown in (c). When there are big 3-d clusters nearby, it may be energetically more favorable for film material adsorbed on the second layer to transfer to a big 3-d cluster nearby. However, if the cluster is not big enough, Ag may go back to the second layer. A schematic is shown in (d). 
with each other, this may be the cause of the existence of Ag clusters instead of random substitutional surface alloy. In particular, we note that since Ag remains either on top or by the side of Mo, the apparent thickness of Ag will show little difference whether such mixing happens or not. This is consistent with the experimental results. Note that for the $\operatorname{Ag} / \operatorname{Pt}\left(\begin{array}{lll}1 & 1 & 1\end{array}\right)$ case, intermixing occurs only [24] when $0<\theta<1$. In the present case of $\mathrm{Ag} / \mathrm{Mo}(111)$, intermixing seems to occur even when the coverage is up to 1.6 TDML. Note that in Fig. 6(a), for curves labeled 160, 170 and 180 , the apparent thickness shows a small rise near $550 \mathrm{~K}$ before the big drop due to 3 -d cluster formation. The small rise suggests that the surface is changing from the $1 \times 6$ and $2 \times 12$ phase separated form into a form of more uniform thickness. Very likely, this phase of more uniform thickness is the ring phase as $550 \mathrm{~K}$ is the temperature ring phases form. Fig. 12 shows schematic drawings of surfaces with and without intermixing and for coverage either below or above one monolayer. In particular, we note that for $\theta>1$, if the density of the immersed 2-d islands is high, there could be a huge number of island ledge sites that presumably have a binding energy close to that of the 3-d clusters.

\section{Conclusion}

A detailed study of the $\mathrm{Ag} / \mathrm{Mo}\left(\begin{array}{lll}1 & 1 & 1\end{array}\right)$ system indicates that $\mathrm{Ag}$ does not induce faceting of Mo(llll). Thus, the "electronegativity correlation" remains intact and it is yet to be answered why or how the electronegativity of an adsorbate influences the surface energy anisotropy. The Ag/ Mo(1 111 ) system grows primarily in the SK mode. However, it has several unusual twists. First, for coverage above the wetting layer, the stability of the metastable 2-d films increases initially instead of simply decreasing monotonically. Also, there are two different types of wetting layers. One has a $1 \times 6$ superstructure while the other thermodynamically more stable one assumes a "ring phase". We propose that the ring phase is due to intermixing of 2-d clusters at the interface. Second, at elevated temperature, 3-d Ag clusters evaporate into a 2-d gas that can reach very high density of 0.8 TDML. Such evaporation is reversible and the 2-d gas condenses back to the 3-d clusters when cooled. Finally, TPA proved to be a very useful tool in keeping track of the real time morphological evolution of these films.

\section{Acknowledgements}

The authors acknowledge helpful discussions with Dr. S.P. Chen of Los Alamos National Laboratory. This work has been supported in part by the National Science Council of the Republic of China under grant no. NSC 89-2112-M-001-030.

\section{References}

[1] T.E. Madey, J. Guan, C.-Z. Dong, S.M. Shivaprasad, Surf. Sci. 287/288 (1993) 826.

[2] C. Zhang, M.A. van Hove, G.A. Somorjai, Surf. Sci. 149 (1985) 326.

[3] J. Guan, R.A. Campbell, T.E. Madey, Surf. Sci. 341 (1995) 311.

[4] K.-J. Song, J.C. Lin, M.Y. Lai, Y.L. Wang, Surf. Sci. 327 (1995) 17.

[5] H.-S. Tao, J.E. Rowe, T.E. Madey, Surf. Sci. 407 (1998) L640.

[6] S.P. Chen, Surf. Sci. 274 (1992) L619.

[7] J.G. Che, C.T. Chan, C.H. Kuo, T.C. Leung, Phys. Rev. Lett. 79 (1997) 4230.

[8] C.H. Nien, T.E. Madey, Y.W. Tai, T.C. Leung, J.G. Che, C.T. Chan, Phys. Rev. B 59 (1999) 10335.

[9] L. Huang, S.J. Chey, J.H. Weaver, Surf. Sci. 416 (1998) L1101.

[10] Z.Y. Zhang, Q. Niu, C.K. Shih, Phys. Rev. Lett. 80 (1998) 5381.

[11] M. Kaliff, G. Comsa, T. Michely, Phys. Rev. Lett. 81 (1998) 1255

[12] S. Tanuma, C.J. Powell, D.R. Penn, Surf. Interface Anal. 11 (1988) 577.

[13] R. Gomer, in: Vu Thien Binh (Ed.), Surface Mobilities on Solid Materials, Plenum, New York, 1983, p. 127.

[14] R.E. Honig, D.A. Kramer, RCA Review 30 (1969) 285.

[15] J. Kolaczkiewicz, E. Bauer, Surf. Sci. 175 (1986) 508.

[16] M. Zinke-Allmang, L.C. Feldman, M.H. Grabow, Surf. Sci. 200 (1988) L427.

[17] H.-J. Gossmann, G.J. Fisanick, Surf. Sci. 244 (1991) L117.

[18] J. Kolaczkiewicz, Surf. Sci. 183 (1987) 251.

[19] A. Zangwill, Physics at Surfaces, Cambridge, New York, 1988, p.118 and references therein.

[20] V.A. Shchukin, D. Bimberg, Rev. Mod. Phys. 71 (1999) 1125. 
[21] K.-O. Ng, D. Vanderbilt, Phys. Rev. B 52 (1995) 2177.

[22] P. Hahn, J. Clabes, M. Henzler, J. Appl. Phys. 51 (1980) 2079.

[23] A.F. Becker, G. Rosenfeld, B. Poelsema, G. Comsa, Phys. Rev. Lett. 70 (1993) 477.
[24] H. Roder, R. Schuster, H. Brune, K. Kern, Phys. Rev. Lett. 71 (1993) 2086.

[25] W.G. Moffat, Handbook of Binary Phase Diagrams, Genium, Schenectady, 1987.

[26] K. Harting, A.P. Janssen, J.A. Venables, Surf. Sci. 74 (1978) 69. 